\title{
On the Covariance Structure of Earnings and Hours Changes
}

\author{
John M. Abowd; David Card \\ Econometrica, Vol. 57, No. 2. (Mar., 1989), pp. 411-445.
}

Stable URL:

http://links.jstor.org/sici?sici=0012-9682\%28198903\%2957\%3A2\%3C411\%3AOTCSOE\%3E2.0.CO\%3B2-U

Econometrica is currently published by The Econometric Society.

Your use of the JSTOR archive indicates your acceptance of JSTOR's Terms and Conditions of Use, available at

http://www.jstor.org/about/terms.html. JSTOR's Terms and Conditions of Use provides, in part, that unless you have obtained prior permission, you may not download an entire issue of a journal or multiple copies of articles, and you may use content in the JSTOR archive only for your personal, non-commercial use.

Please contact the publisher regarding any further use of this work. Publisher contact information may be obtained at http://www.jstor.org/journals/econosoc.html.

Each copy of any part of a JSTOR transmission must contain the same copyright notice that appears on the screen or printed page of such transmission.

The JSTOR Archive is a trusted digital repository providing for long-term preservation and access to leading academic journals and scholarly literature from around the world. The Archive is supported by libraries, scholarly societies, publishers, and foundations. It is an initiative of JSTOR, a not-for-profit organization with a mission to help the scholarly community take advantage of advances in technology. For more information regarding JSTOR, please contact support@jstor.org. 


\title{
ON THE COVARIANCE STRUCTURE OF EARNINGS AND HOURS CHANGES
}

\author{
By John M. ABowd and David CARD ${ }^{1}$
}

\begin{abstract}
This paper presents an empirical analysis of individual earnings and hours data from three different longitudinal surveys. In the first part of the paper we catalog the main features of the covariance structure of earnings and hours changes. We find that this structure is very similar across data sets, and may be adequately summarized by a simple components-of-variance model, consisting of (i) serially uncorrelated measurement error, (ii) a shared component of earnings and hours with a second-order moving average covariance structure, and (iii) a nonstationary component that affects only the variances and contemporaneous covariances of earnings and hours. In the second part of the paper we offer an interpretation of this model in terms of a simple life-cycle labor supply model. On the assumption that we can identify individual productivity growth with the shared component of earnings and hours variation, we obtain estimates of the intertemporal substitution elasticity. The results are not favorable to the life-cycle model: most of the covariation of earnings and hours occurs at fixed hourly wage rates.
\end{abstract}

KEYwORDS: Components-of-variance models, lifecycle labor supply, measurement error, intertemporal substitution.

RECENT STUDIES OF INDIVIDUAL EARNINGS and hours data focus on the contemporaneous correlation between hours of work and average hourly earnings. ${ }^{2}$ This focus arises naturally from the life-cycle labor supply model, which explains changes in individual hours by changes in the value of work and unanticipated changes in wealth. ${ }^{3}$ Nonetheless, there is widespread agreement that most of the observed variation in hours over time is not explained by contemporaneous movements in wages. ${ }^{4}$ On one hand, the cross-sectional correlation between percentage changes in annual hours and percentage changes in average hourly earnings is apparently dominated by measurement error. ${ }^{5}$ On the other hand, state-of-the-art estimates of the life-cycle labor supply model yield small and often statistically insignificant elasticities between hours variation and wage movements. ${ }^{6}$

${ }^{1}$ We are grateful to Gary Chamberlain, Zvi Griliches, Whitney Newey, and Robert Topel for comments and suggestions. We thank the Department of Economics at the Massachusetts Institute of Technology for their hospitality while the first draft of this paper was written.

${ }^{2}$ See for example Ashenfelter and Ham (1977), MaCurdy (1981), Altonji (1986), and the surveys by Killingsworth (1983) and Pencavel (1986).

${ }^{3}$ The life-cycle labor supply model is described in Ghez and Becker (1975) and Heckman (1976). Browning, Deaton, and Irish (1985) provide a useful summary of the theory of consumer behavior over time.

${ }^{4}$ Pencavel (1986) concludes that "... the focus of most economists' research [on labor supply] has been on a behavioral response that for men appears to be of a relatively small order of magnitude."

${ }^{5}$ Altonji (1986) compares the correlation between changes in hours and changes in average hourly earnings (about -.35 in his sample and in three of the four samples used in this paper) with the correlation between changes in hours and changes in reported wage rates of hourly rated workers (about .01 in Altonji's sample). Altonji interprets this difference as evidence that measurement error in hours induces a strong negative correlation between changes in hours and changes in average hourly earnings.

${ }^{6}$ MaCurdy (1981) and Altonji (1986) both arrive at estimates of the intertemporal substitution elasticity in the neighborhood of .10 to .40 . 
In this paper, we present a more general analysis of the relation between movements in earnings and movements in hours over time. ${ }^{7}$ Using data from three different longitudinal surveys, we first catalogue the main features of the covariance structure of earnings and hours changes. We find that a relatively simple components-of-variance model summarizes the data from all three surveys. We then compare this covariance structure to the structure implied by a simple version of the life-cycle labor supply model. According to the life-cycle model, changes in individual productivity lead to changes in earnings and hours, with a larger effect on earnings than hours. In contrast, our empirical results suggest that the main source of shared variation in earnings and hours represents changes in hours at fixed hourly wage rates.

In the first section of the paper, we describe the covariance matrix of changes in annual earnings and annual hours of work for male household heads in four data sets: two samples from the Panel Study of Income Dynamics (PSID); a sample of older men from the National Longitudinal Survey of Men 49-59 (NLS); and a sample from the control group of the Seattle and Denver Income Maintenance Experiment (SIME/DIME). We then test for parsimonious representations of the covariance matrices from these surveys. In all four samples we find that changes in earnings and changes in hours are uncorrelated with lagged changes more than two periods apart. We also find strong evidence of nonstationarity in the covariances of earnings and hours.

We go on to examine some relatively simple models for the covariance structure of earnings and hours changes. The first model is one of pure measurement error, in which changes in earnings and hours result from random respondent and interviewer errors. While this simple model captures some of the main features of the data, it is overly restrictive in several important dimensions. The next model adds a single component of variance that enters with different coefficients in earnings and hours. This model provides a significantly better fit to the covariance matrices of earnings and hours changes, but is still overly restrictive. The final model we present adds a time-varying component to the variances of changes in earnings and hours. This relatively simple three-component model provides a statistically acceptable description of the covariances from all three data sources.

In the second section of the paper we present a model of the covariance structure of earnings and hours based on the life-cycle labor supply model. This model implies a simple structure in which individual productivity enters as a common component of variance in earnings and hours. Other components include measurement error, changes in tastes for leisure, and unanticipated changes in the marginal utility of consumption. The key implication of the life-cycle labor supply model is that changes in individual productivity influence earnings more than hours. The estimated covariance structure of all four samples,

\footnotetext{
${ }^{7}$ Our empirical analysis is closely related to work by Lillard and Weiss (1979), Hause (1980), and MaCurdy (1982). These authors all model the serial correlation structure of earnings in longitudinal data. We extend the analysis to the bivariate process of earnings and hours.
} 
however, suggests that the common component of earnings and hours variation affects the two variables proportionally. This leads us to conclude that labor supply responses to individual productivity variation are not an important source of variation in individual labor market outcomes. Rather, most changes in earnings and hours occur at fixed wage rates.

\section{DATA DESCRIPTION}

The earnings and hours data in this paper are drawn from the Panel Study of Income Dynamics, the National Longitudinal Survey of Men 45-59, and the nonexperimental families in the Seattle and Denver Income Maintenance Experiment. For the PSID we used the Wave XIII Merged Individual Tape (Survey Research Center, 1972 and 1981). For the NLS we used the Public Use Tape Release 75A (Center for Human Resource Research, 1977 and 1981). For the SIME/DIME we used the Work Impact Dual Head of Household File (SRI International, 1983a). Appendix B describes the procedures we used to obtain each sample.

From the PSID we have selected two alternative samples of male household heads. Our first sample consists of men who were heads of household in every year from 1969 to 1979 (the third through thirteenth waves of the survey), who were between the ages of 21 and 64 in each year, and who reported positive earnings and hours in each year. This sample contains a total of 1448 individuals. Sample average annual hours and hourly wage rates, as well as averages of the changes in the logarithms of annual earnings and hours, are reported in Table I.

Like the other two surveys used in this paper the PSID survey overrepresents low income and nonwhite households. ${ }^{8}$ In our sample of male heads with positive earnings and hours in every year, the fraction of nonwhites is 27.3 percent. At the suggestion of the editor and referees, we have therefore drawn a second sample of the PSID which excludes individuals from the subset of the PSID drawn from Survey of Economic Opportunity (SEO). The characteristics of this alternative sample are reported in the right-hand columns of Table I. As expected, this sample of 991 males contains many fewer nonwhites, and has higher average hourly earnings. Average annual hours, on the other hand, are very similar between the overall PSID sample and the subsample which excludes the SEO.

From the NLS we have selected 1318 men who were less than 65 years old in 1975 and who reported nonzero earnings and hours in each of the survey years $1966,1967,1969,1971,1973$, and $1975 .{ }^{9}$ Table II summarizes the characteristics

\footnotetext{
${ }^{8}$ Information on the sampling frames of the three surveys are contained in Survey Research Center (1972), Center for Human Resource Research (1977), and SRI International (1983b).

${ }^{9}$ The $1969,71,73$, and 75 NLS surveys were administered in July through October of the survey years, and asked questions on earnings and hours in the previous twelve months. Earlier surveys $(1966,1967)$ collected earnings information for the previous calendar year. Hours information is for calendar year 1965 in the 1966 survey and for the previous twelve months in the 1967 survey. Unlike the PSID survey, the NLS collects no auxilliary information on overtime hours or hours of work on secondary jobs. For this and perhaps other reasons a large fraction (approximately 30 percent) of NLS respondents report exactly 2,000 hours per year in any survey year.
} 
of this sample. The fraction of nonwhites in the NLS sample is similar to that in the overall PSID sample, and much higher than the fraction in the PSID subsample without the SEO. It is important to keep in mind that all but the first two waves of the NLS survey were administered biennially. As a consequence, four of the five available changes in earnings and hours for this sample refer to changes in annual totals over two-year intervals.

From the control group of the Seattle-Denver Income Experiment our sample consists of 560 male heads of dual-headed households. ${ }^{10}$ These male heads were between 21 and 64 years of age during the first four years of the experiment, and reported nonzero earnings and hours in each of the first eight experimental half-year periods. Demographic characteristics and average hourly wage rates and hours for the SIME/DIME sample are recorded in Table III. Labor market information in the income maintenance experiments was collected quarterly and aggregated into six-month intervals by the experiments' contractors. Hours of work and changes in earnings and hours for the SIME/DIME sample therefore refer to labor market outcomes in six-month periods. Since enrollment into the experiments was conducted over a two year interval, the six-month experimental periods refer to different periods of calendar time for different sample members. Labor market outcomes in the first experimental period, for example, refer to data for calendar periods between early 1971 and late 1972.

By design, the income experiments heavily oversampled low income and nonwhite households. The fraction of nonwhites in our SIME/DIME sample is consequently higher than the fraction in either the NLS or overall PSID samples. Average hourly earnings and annual hours are also somewhat lower for the SIME/DIME sample than for any of the other three samples.

The complete covariance matrices of the changes in the logarithms of annual earnings and annual hours for the four samples are recorded in Tables IV-VII. In order to control for differences in labor force experience within and between the samples, we have computed these covariances using the residuals from unrestricted multivariate regressions of changes in earnings and hours on time period indicator variables and potential experience (age minus education minus five). As it happens, the characteristics of the data are not significantly affected by this adjustment, since the explanatory power of the experience regressions is negligible in all four samples. The covariances of the changes in earnings and hours with potential experience are recorded in the final row of each table.

To control for the fact that the SIME/DIME data are drawn from different calendar periods, depending on the date of assignment into the experiment, changes in earnings and hours from the SIME/DIME sample are regression adjusted using potential experience and a series of indicator variables for monthof-assignment into the experiment. In none of the sixteen regressions for the eight changes in earnings and hours were these indicator variables jointly statistically significant at conventional significance levels.

\footnotetext{
${ }^{10}$ The SIME/DIME survey contains detailed questions on overtime and secondary jobs, and is conceptually more similar to the PSID survey than the NLS survey. See Appendix B.
} 
TABLE I

Characteristics of the PSID Sample of Male Household Heads

\begin{tabular}{|c|c|c|c|c|c|c|}
\hline & & \multirow[b]{2}{*}{ Year ${ }^{b}$} & \multicolumn{2}{|c|}{ SEO Included } & \multicolumn{2}{|c|}{ SEO Excluded ${ }^{\mathbf{a}}$} \\
\hline & & & $\begin{array}{c}\text { Average } \\
\text { Hourly } \\
\text { Earnings }\end{array}$ & $\begin{array}{c}\text { Average } \\
\text { Annual } \\
\text { Hours }\end{array}$ & $\begin{array}{c}\text { Average } \\
\text { Hourly } \\
\text { Earnings }\end{array}$ & $\begin{array}{c}\text { Average } \\
\text { Annual } \\
\text { Hours }\end{array}$ \\
\hline \multirow[t]{12}{*}{1.} & Annual Hours and Average & 1969 & 3.62 & 2308 & 4.00 & 2291 \\
\hline & Hourly Earnings (1967 dollars) & 1970 & 3.71 & 2276 & 4.15 & 2266 \\
\hline & & 1971 & 3.85 & 2266 & 4.24 & 2290 \\
\hline & & 1972 & 4.00 & 2302 & 4.47 & 2313 \\
\hline & & 1973 & 4.13 & 2324 & 4.59 & 2319 \\
\hline & & 1974 & 4.10 & 2246 & 4.54 & 2268 \\
\hline & & 1975 & 4.02 & 2220 & 4.46 & 2234 \\
\hline & & 1976 & 4.19 & 2231 & 4.57 & 2246 \\
\hline & & 1977 & 4.26 & 2236 & 4.70 & 2253 \\
\hline & & 1978 & 4.26 & 2244 & 4.91 & 2239 \\
\hline & & 1979 & 4.25 & 2186 & 4.93 & 2192 \\
\hline & & Change & $\begin{array}{l}\text { Change in } \\
\text { Earnings }\end{array}$ & $\begin{array}{l}\text { Change } \\
\text { in Hours }\end{array}$ & $\begin{array}{l}\text { Change in } \\
\text { Earnings }\end{array}$ & $\begin{array}{l}\text { Change } \\
\text { in Hours }\end{array}$ \\
\hline & Changes in Log Real Annual & $1969-70$ & 2.5 & -0.8 & 3.6 & -.6 \\
\hline & Earnings of Log Annual & $1970-71$ & 3.0 & -0.3 & 2.0 & .6 \\
\hline & Hours $(\times 100)$ & $1971-72$ & 6.9 & 2.0 & 9.0 & 2.6 \\
\hline & & $1972-73$ & 4.7 & 1.9 & 3.0 & .5 \\
\hline & & $1973-74$ & -5.5 & -4.1 & -3.7 & -2.3 \\
\hline & & $1974-75$ & -4.2 & -2.4 & -4.3 & -2.5 \\
\hline & & $1975-76$ & 4.1 & 0.6 & 2.0 & .1 \\
\hline & & $1976-77$ & 2.5 & 0.3 & 4.7 & .7 \\
\hline & & $1977-78$ & 0.2 & 0.5 & -1.3 & -2.0 \\
\hline & & $1978-79$ & -5.5 & -4.2 & -5.7 & -3.5 \\
\hline \multicolumn{7}{|c|}{ 3. Demographic Characteristics } \\
\hline & Average Age in 1969 & & 35.8 & & 36.7 & \\
\hline & Percent Nonwhite & & 27.3 & & 8.2 & \\
\hline & Average Potential & & 18.9 & & 19.1 & \\
\hline & Experience in 1969 & & & & & \\
\hline & Sample Size ${ }^{c}$ & & 1448 & & 991 & \\
\hline
\end{tabular}

${ }^{\text {a }}$ Sample members from the Survey of Economic Opportunity (SEO) are excluded.

${ }^{b}$ Data are for the calendar years listed.

'Sample excludes observations with average hourly earnings greater than $\$ 100 /$ hour, or annual hours greater than 4680 .

Table IV contains the experience-adjusted covariances and correlations of changes in the logarithms of annual earnings and annual hours for our overall sample of household heads from the PSID. The covariances and their associated standard errors are presented below the diagonal of the table, while the correlation coefficients are presented above the diagonal. The table is arranged with the 10 changes in log earnings (denoted by $\Delta \log g$ ) first, and the 10 changes in $\log$ hours (denoted by $\Delta \log h$ ) following.

Looking first at the diagonal elements of Table IV, the cross-sectional variation in percentage changes in annual earnings and hours is large: the standard deviation of the change in the logarithm of earnings is at least 35 percent, while the standard deviation of the change in the logarithm of annual hours is at least 25 percent. The variances of changes in annual earnings and hours also vary over 
TABLE II

Characteristic of the NLS SAmple of Older Men

\begin{tabular}{lccr}
\hline \hline & & $\begin{array}{c}\text { Average } \\
\text { Hourly } \\
\text { Earnings }\end{array}$ & $\begin{array}{c}\text { Average } \\
\text { Annual } \\
\text { Hours }\end{array}$ \\
\hline 1. Annual Hours and Average & 1966 & 3.50 & 2209 \\
$\quad$ Hourly Earnings (1967 dollars) & 1967 & 3.46 & 2190 \\
& 1969 & 3.55 & 2190 \\
& 1971 & 3.66 & 2161 \\
& 1973 & 3.63 & 2160 \\
& 1975 & 3.50 & 2003 \\
\hline & & Change & Change \\
& Change & in Earnings & in Hours \\
& $1966-67$ & 4.5 & 0.0 \\
2. Changes in Log Real Annual & $1967-69$ & 4.0 & 0.0 \\
$\quad$ Earnings and Log Annual & $1969-71$ & 3.1 & -0.1 \\
$\quad$ Hours ( $\times 100$ ) & $1971-73$ & -0.2 & -1.5 \\
& $1973-75$ & -16.8 & -11.6 \\
3. Demographic Characteristics & & & \\
$\quad$ Average Age in 1969 & & 49.2 & \\
$\quad$ Percent Nonwhite & & 29.8 & \\
$\quad$ Average Potential & & 34.4 & \\
$\quad$ Experience in 1969 & & 1318 & \\
4. Sample Size & & & \\
\hline
\end{tabular}

${ }^{a}$ Data are for twelve-month periods preceeding the interview date (except 1966 and 1967; see footnote 9).

${ }^{\mathrm{b}}$ Changes in earnings and hours over the two year intervals are not at annual rates.

time. Cross-sectional dispersion in earnings and hours growth was relatively small in 1972-73 and 1973-74, and relatively large in 1975-76.

Turning to the first-order autocovariances, which are displayed directly below the diagonal, it is clear that consecutive changes in individual earnings and hours are strongly negatively correlated. The average covariance of consecutive changes in earnings is -.060 : the average first-order correlation coefficient is -.34 . Similarly, the average first-order autocovariance of the change in hours is - .061, with an average first-order autocorrelation coefficient of -.34 .

The cross-covariances between changes in earnings and changes in hours are displayed in the lower left-hand block of the table. Contemporaneous changes in earnings and hours are significantly positively correlated: the average covariance is .073 , with an average correlation coefficient of .50. Since the logarithm of average hourly earnings is simply the difference in logarithms of annual earnings and annual hours, the covariance between changes in hours and changes in average hourly earnings is the difference between the covariance of earnings and hours and the variance of hours. This difference is uniformly negative in Table IV, implying that changes in hours and changes in average hourly earnings are negatively correlated.

A final feature of the covariance matrix in Table IV is the absence of any large or statistically significant covariances at lags greater than two years. Year-to-year changes in earnings and hours more than two years apart are approximately 
TABLE III

Characteristics of the SIME / DIME Sample of Male-Heads of Dual-Headed Households

\begin{tabular}{lccr}
\hline \hline & $\begin{array}{c}\text { Experimental } \\
\text { Period }^{2}\end{array}$ & $\begin{array}{c}\text { Average } \\
\text { Hourly } \\
\text { Earnings }\end{array}$ & $\begin{array}{r}\text { Average } \\
\text { Annual } \\
\text { Hours }\end{array}$ \\
\hline 1. Annual Hours and Average & 1 & 3.47 & 2093 \\
$\quad$ Hourly Earnings (1971 dollars) & 2 & 3.53 & 2098 \\
& 3 & 3.63 & 2087 \\
& 4 & 3.73 & 2117 \\
& 5 & 3.83 & 2135 \\
& 6 & 3.88 & 2104 \\
& 7 & 3.88 & 2131 \\
& 8 & 3.85 & 2074 \\
& 9 & 3.88 & 2059 \\
\hline 2. Changes in Log Real Semi-Annual & & Change & Change \\
Earnings and Log Semi-Annual & Change & in Earnings & Hours \\
Hours ( $\times 100$ ) & $1-2$ & 1.2 & 3.9 \\
& $2-3$ & -1.3 & 1.0 \\
& $3-4$ & 2.8 & 5.8 \\
& $4-5$ & 1.6 & 4.0 \\
& $5-6$ & -1.3 & 0.2 \\
& $6-7$ & 0.7 & 0.6 \\
& $7-8$ & -3.5 & -4.5 \\
& $8-9$ & -3.0 & -2.7 \\
3. Demographic Characteristics & & & \\
Average Age at start of experiment & & 34.7 & \\
Percent Nonwhite & & 48.8 & \\
Average Potential Experience & & 18.2 & \\
at start of experiment & & $560^{\mathrm{c}}$ & \\
4. Sample Size & & & \\
\hline
\end{tabular}

${ }^{\text {a }}$ Data are for six-month periods following assignment into the income experiment. Annual hours are estimated as twice the semiannual average.

${ }^{b}$ The changes in earnings and hours between consecutive six-month intervals are not at annual rates.

${ }^{c}$ Sample excludes individuals with more than 2500 hours of work in any half-year period.

uncorrelated. This suggests that changes in earnings and changes in hours may be adequately summarized by a (possibly nonstationary) bivariate second order moving average (MA(2)) process.

Table $\mathrm{V}$ displays the equivalent covariance matrix of earnings and hours changes for the PSID sample without the SEO subsample. The main features of the table are similar to those in Table IV, although the variances and covariances are somewhat smaller in absolute value when the SEO group is excluded. The average first-order autocorrelations of earnings and hours changes are also slightly lower in Table V $(-.29$ and -.33 , respectively). Again, the covariance between earnings and hours changes is positive but smaller than the variance of hours changes, implying that changes in hours and changes in average hourly earnings are negatively correlated. As in Table IV, few of the covariances between earnings and hours changes more than two years apart are large or statistically different from zero. Finally, the pattern of nonstationarity in the variances and covariances is similar between the overall PSID sample and the sample with the SEO excluded. 
TABLE IV

Covariances and Correlations ${ }^{a}$ Between Experience Adjusted Changes in Earnings and Hours: PSID Males 1969 To $1979^{\text {b }}$ (Standard Errors in Parentheses)

\begin{tabular}{|c|c|c|c|c|c|c|c|c|c|c|c|c|c|c|c|c|c|c|c|c|}
\hline with: & $\begin{array}{c}(1) \\
\Delta \log g \\
69-70\end{array}$ & $\begin{array}{c}(2) \\
\Delta \log g \\
70-71\end{array}$ & $\begin{array}{c}(3) \\
\Delta \log g \\
71-72\end{array}$ & $\begin{array}{l}(4) \\
\Delta \log g \\
72-73\end{array}$ & $\begin{array}{c}(5) \\
\Delta \log g \\
73-74\end{array}$ & $\begin{array}{c}(6) \\
\Delta \log g \\
74-75\end{array}$ & $\begin{array}{c}(7) \\
\Delta \log g \\
75-76\end{array}$ & $\begin{array}{c}(8) \\
\Delta \log g \\
76-77\end{array}$ & $\begin{array}{c}(9) \\
\Delta \log g \\
77-78\end{array}$ & $\begin{array}{c}\text { Covari } \\
(10) \\
\Delta \log g \\
78-79\end{array}$ & $\begin{array}{c}\text { nce of: } \\
\quad(11) \\
\Delta \log h \\
69-70\end{array}$ & $\begin{array}{c}(12) \\
\Delta \log h \\
70-71\end{array}$ & $\begin{array}{c}(13) \\
\Delta \log h \\
71-72\end{array}$ & $\begin{array}{c}(14) \\
\Delta \log h \\
72-73\end{array}$ & $\begin{array}{c}(15) \\
\Delta \log h \\
73-74\end{array}$ & $\begin{array}{c}(16) \\
\Delta \log h \\
74-75\end{array}$ & $\begin{array}{c}(17) \\
\Delta \log h \\
75-76\end{array}$ & $\begin{array}{c}(18) \\
\Delta \log h \\
76-77\end{array}$ & $\begin{array}{c}(19) \\
\Delta \log h \\
77-78\end{array}$ & $\begin{array}{c}(20) \\
\Delta \log h \\
78-79\end{array}$ \\
\hline 1. $\begin{array}{c}\Delta \log g \\
69-70\end{array}$ & $\begin{array}{c}.161 \\
(.018)\end{array}$ & -.23 & -.04 & .00 & .02 & .03 & -.04 & -.01 & -.01 & .00 & .48 & -.17 & -.01 & .07 & -.01 & .05 & -.06 & .02 & -.01 & .01 \\
\hline 2. $\begin{array}{l}\Delta \log g \\
70-71\end{array}$ & $\begin{array}{r}-.036 \\
(.013)\end{array}$ & $\begin{array}{c}.158 \\
(.019)\end{array}$ & -.39 & -.02 & .00 & .03 & -.04 & .00 & .04 & -.02 & -.09 & .53 & -.23 & .01 & -.03 & .02 & -.04 & .03 & .00 & -.00 \\
\hline 3. $\begin{array}{l}\Delta \log g \\
71-72\end{array}$ & $\begin{array}{r}-.007 \\
(.007)\end{array}$ & $\begin{array}{r}-.064 \\
(.013)\end{array}$ & $\begin{array}{c}.170 \\
(.019)\end{array}$ & -.41 & -.05 & -.06 & .05 & .05 & -.03 & .01 & -.01 & -.19 & .56 & -.20 & -.10 & .00 & .02 & -.01 & .03 & -.02 \\
\hline 4. $\begin{array}{ll}\Delta \log g \\
72-73\end{array}$ & $\begin{array}{c}.000 \\
(.006)\end{array}$ & $\begin{array}{c}-.002 \\
(.008)\end{array}$ & $\begin{array}{r}-.062 \\
(.013)\end{array}$ & $\begin{array}{c}.134 \\
(.014)\end{array}$ & -.28 & -.07 & .00 & .07 & .04 & .00 & .02 & -.04 & -.17 & .38 & .04 & -.07 & -.02 & -.02 & .03 & .02 \\
\hline 5. $\frac{\Delta \log g}{73-74}$ & $\begin{array}{l}.003 \\
(.004)\end{array}$ & $\begin{array}{c}.000 \\
(.005)\end{array}$ & $\begin{array}{r}-.007 \\
(.005)\end{array}$ & $\begin{array}{c}-.036 \\
(.007)\end{array}$ & $\begin{array}{l}.129 \\
(.016)\end{array}$ & -.35 & -.01 & -.06 & -.01 & .00 & -.01 & .02 & -.06 & .05 & .37 & -.15 & -.02 & .00 & -.05 & -.01 \\
\hline 6. $\frac{\Delta \log g}{74-75}$ & $\begin{array}{c}.005 \\
(.005)\end{array}$ & $\begin{array}{c}.005 \\
(.005)\end{array}$ & $\begin{array}{r}-.010 \\
(.007)\end{array}$ & $\begin{array}{c}-.011 \\
(.006)\end{array}$ & $\begin{array}{r}-.054 \\
(.015)\end{array}$ & $\begin{array}{c}.181 \\
(.021)\end{array}$ & -.44 & -.06 & -.03 & .00 & -.02 & .02 & -.06 & -.03 & -.07 & .51 & -.30 & -.04 & .02 & -.01 \\
\hline 7. $\begin{array}{c}\Delta \log g \\
75-76\end{array}$ & $\begin{array}{r}-.007 \\
(.006)\end{array}$ & $\begin{array}{r}-.007 \\
(.005)\end{array}$ & $\begin{array}{c}.010 \\
(.008)\end{array}$ & $\begin{array}{c}.000 \\
(.008)\end{array}$ & $\begin{array}{r}-.001 \\
(.006)\end{array}$ & $\begin{array}{r}-.088 \\
(.015)\end{array}$ & $\begin{array}{c}.224 \\
(.032)\end{array}$ & -.42 & -.07 & .00 & .03 & -.05 & .06 & -.02 & -.06 & -.20 & .59 & -.25 & -.08 & -.03 \\
\hline 8. $\begin{array}{c}\Delta \log g \\
76-77\end{array}$ & $\begin{array}{r}-.002 \\
(.005)\end{array}$ & $\begin{array}{c}.000 \\
(.007)\end{array}$ & $\begin{array}{c}.009 \\
(.008)\end{array}$ & $\begin{array}{c}.011 \\
(.008)\end{array}$ & $\begin{array}{c}-.010 \\
(.006)\end{array}$ & $\begin{array}{c}-.011 \\
(.007)\end{array}$ & $\begin{array}{c}-.087 \\
(.028)\end{array}$ & $\begin{array}{c}.194 \\
(.034)\end{array}$ & -.34 & .01 & -.02 & .03 & -.02 & .05 & -.09 & .02 & -.28 & .58 & -.20 & -.02 \\
\hline 9. $\begin{array}{c}\Delta \log g \\
77-78\end{array}$ & $\begin{array}{c}-.003 \\
(.007)\end{array}$ & $\begin{array}{c}.006 \\
(.007)\end{array}$ & $\begin{array}{r}-.005 \\
(.008)\end{array}$ & $\begin{array}{c}.006 \\
(.007)\end{array}$ & $\begin{array}{c}-.001 \\
(.006)\end{array}$ & $\begin{array}{c}-.006 \\
(.007)\end{array}$ & $\begin{array}{c}-.015 \\
(.008)\end{array}$ & $\begin{array}{c}-.066 \\
(.018)\end{array}$ & $\begin{array}{c}.193 \\
(.027)\end{array}$ & -.24 & -.01 & .04 & -.01 & -.07 & .02 & -.09 & .02 & -.20 & .55 & .03 \\
\hline
\end{tabular}




\begin{tabular}{|c|c|c|c|c|c|c|c|c|c|c|c|c|c|c|c|c|c|c|c|c|c|}
\hline & $\begin{array}{l}\Delta \log g \\
78-79\end{array}$ & $\begin{array}{c}.000 \\
(.004)\end{array}$ & $\begin{array}{c}-.003 \\
(.005)\end{array}$ & $\begin{array}{c}.002 \\
(.005)\end{array}$ & $\begin{array}{c}.000 \\
(.005)\end{array}$ & $\begin{array}{c}.000 \\
(.006)\end{array}$ & $\begin{array}{c}.000 \\
(.006)\end{array}$ & $\begin{array}{c}.001 \\
(.006)\end{array}$ & $\begin{array}{c}.003 \\
(.010)\end{array}$ & $\begin{array}{r}-.044 \\
(.013)\end{array}$ & $\begin{array}{c}.179 \\
(.020)\end{array}$ & -.05 & -.01 & .02 & .04 & .02 & .03 & -.04 & .05 & -.04 & 48 \\
\hline & $\begin{array}{l}\Delta \log \\
69-7\end{array}$ & $\begin{array}{l}.067 \\
(.014)\end{array}$ & $\begin{array}{r}-.012 \\
(.010)\end{array}$ & $\begin{array}{r}-.001 \\
(.007)\end{array}$ & $\begin{array}{l}.002 \\
(.004)\end{array}$ & $\begin{array}{r}-.002 \\
(.003)\end{array}$ & $\begin{array}{r}-.003 \\
(.005)\end{array}$ & $\begin{array}{c}.004 \\
(.006)\end{array}$ & $\begin{array}{c}-.004 \\
(.005)\end{array}$ & $\begin{array}{c}-.001 \\
(.004)\end{array}$ & $\begin{array}{r}-.007 \\
(.004)\end{array}$ & $\begin{array}{c}.118 \\
(.015)\end{array}$ & -.34 & -.01 & .00 & .06 & -.05 & -.02 & .02 & -.01 & -.01 \\
\hline & & $\begin{array}{r}-.022 \\
(.011)\end{array}$ & $\begin{array}{c}.071 \\
(.015)\end{array}$ & $\begin{array}{r}-.026 \\
(.011)\end{array}$ & $\begin{array}{r}-.005 \\
(.007)\end{array}$ & $\begin{array}{l}.002 \\
(.003)\end{array}$ & $\begin{array}{c}.003 \\
(.005)\end{array}$ & $\begin{array}{r}-.009 \\
(.006)\end{array}$ & $\begin{array}{c}.004 \\
(.005)\end{array}$ & $\begin{array}{c}.005 \\
(.005)\end{array}$ & $\begin{array}{c}-.001 \\
(.004)\end{array}$ & $\begin{array}{c}-.040 \\
(.010)\end{array}$ & $\begin{array}{l}.115 \\
(.015)\end{array}$ & -.41 & -.05 & -.06 & .05 & .00 & -.01 & .00 & .04 \\
\hline & $\begin{array}{l}\Delta \log h \\
71-72\end{array}$ & & & & & $\begin{array}{r}-.007 \\
(.004)\end{array}$ & $\begin{array}{r}-.009 \\
(.004)\end{array}$ & & $\begin{array}{c}-.003 \\
(.005)\end{array}$ & & & $\begin{array}{r}-.002 \\
(.007)\end{array}$ & $\begin{array}{r}-.048 \\
(.010)\end{array}$ & $\begin{array}{c}.118 \\
(.015)\end{array}$ & -.37 & -.10 & -.04 & .02 & -.01 & .02 & -.05 \\
\hline 4 & $\begin{array}{l}\Delta \mathrm{lo} \\
72-\end{array}$ & & & & & & $\begin{array}{r}-.003 \\
(.004)\end{array}$ & $\begin{array}{c}-.002 \\
(.005)\end{array}$ & & $(.00$ & $\begin{array}{l}.005 \\
(.004)\end{array}$ & $\begin{array}{c}.000 \\
(.003)\end{array}$ & $\begin{array}{c}-.005 \\
(.005)\end{array}$ & $\begin{array}{r}-.036 \\
(.010)\end{array}$ & $\begin{array}{c}.080 \\
(.011)\end{array}$ & -.20 & -.01 & -.08 & .07 & -.06 & -.02 \\
\hline & & & $\begin{array}{c}-.003 \\
(.003)\end{array}$ & $\begin{array}{c}-.011 \\
(.006)\end{array}$ & & $\begin{array}{c}.036 \\
(.007)\end{array}$ & $\begin{array}{c}-.008 \\
(.008)\end{array}$ & $\begin{array}{r}-.008 \\
(.007)\end{array}$ & $\begin{array}{c}-.010 \\
(.005)\end{array}$ & & $\begin{array}{c}.002 \\
(.004)\end{array}$ & $\begin{array}{c}.006 \\
(.004)\end{array}$ & $\begin{array}{r}-.006 \\
(.004)\end{array}$ & $\begin{array}{c}-.010 \\
(.003)\end{array}$ & $\begin{array}{c}-.016 \\
(.003)\end{array}$ & $\begin{array}{c}.073 \\
(.007)\end{array}$ & -.34 & -.06 & -.03 & -.02 & .01 \\
\hline & & & & & $\begin{array}{r}-.009 \\
(.004)\end{array}$ & & $\begin{array}{c}.072 \\
(.013)\end{array}$ & $\begin{array}{c}-.031 \\
(.012)\end{array}$ & $\begin{array}{c}.002 \\
(.008)\end{array}$ & $\begin{array}{c}-.013 \\
(.009)\end{array}$ & $\begin{array}{c}.004 \\
(.006)\end{array}$ & $\begin{array}{c}-.006 \\
(.004)\end{array}$ & $\begin{array}{c}.006 \\
(.004)\end{array}$ & $\begin{array}{c}-.005 \\
(.003)\end{array}$ & $\begin{array}{c}-.001 \\
(.003)\end{array}$ & $\begin{array}{c}-.030 \\
(.005)\end{array}$ & $\begin{array}{c}.110 \\
(.013)\end{array}$ & -.45 & -.08 & -.02 & -.02 \\
\hline & & $\begin{array}{r}-.009 \\
(.004)\end{array}$ & $\begin{array}{r}-.006 \\
(.004)\end{array}$ & $\begin{array}{c}.003 \\
(.005)\end{array}$ & $\begin{array}{r}-.002 \\
(.005)\end{array}$ & $\begin{array}{r}-.002 \\
(.004)\end{array}$ & $\begin{array}{r}-.048 \\
(.012)\end{array}$ & $\begin{array}{l}.106 \\
(.022)\end{array}$ & $\begin{array}{c}-.047 \\
(.020)\end{array}$ & $\begin{array}{l}.003 \\
(.010)\end{array}$ & $\begin{array}{r}-.007 \\
(.006)\end{array}$ & $\begin{array}{c}-.002 \\
(.005)\end{array}$ & $\begin{array}{c}.000 \\
(.004)\end{array}$ & $\begin{array}{c}.003 \\
(.003)\end{array}$ & $\begin{array}{r}-.009 \\
(.003)\end{array}$ & $\begin{array}{c}-.006 \\
(.004)\end{array}$ & $\begin{array}{r}-.058 \\
(.013)\end{array}$ & $\begin{array}{l}.147 \\
(.021)\end{array}$ & -.44 & -.04 & -.02 \\
\hline & & $\begin{array}{c}.004 \\
(.004)\end{array}$ & $\begin{array}{c}.004 \\
(.006)\end{array}$ & $\begin{array}{r}-.002 \\
(.005)\end{array}$ & $\begin{array}{r}-.003 \\
(.006)\end{array}$ & $\begin{array}{l}.000 \\
(.005)\end{array}$ & $\begin{array}{r}-.006 \\
(.006)\end{array}$ & $\begin{array}{r}-.044 \\
(.023)\end{array}$ & $\begin{array}{c}.097 \\
(.027)\end{array}$ & $\begin{array}{c}-.033 \\
(.014)\end{array}$ & $\begin{array}{c}.008 \\
(.008)\end{array}$ & $\begin{array}{c}.003 \\
(.005)\end{array}$ & $\begin{array}{r}-.002 \\
(.004)\end{array}$ & $\begin{array}{c}-.001 \\
(.004)\end{array}$ & $\begin{array}{c}.008 \\
(.005)\end{array}$ & $\begin{array}{c}-.003 \\
(.005)\end{array}$ & $\begin{array}{r}-.010 \\
(.006)\end{array}$ & $\begin{array}{r}-.064 \\
(.018)\end{array}$ & $\begin{array}{c}.143 \\
(.024)\end{array}$ & -.38 & -.03 \\
\hline & & $\begin{array}{r}-.002 \\
(.004)\end{array}$ & $\begin{array}{c}.000 \\
(.005)\end{array}$ & $\begin{array}{c}.005 \\
(.005)\end{array}$ & $\begin{array}{c}.005 \\
(.005)\end{array}$ & $\begin{array}{r}-.007 \\
(.004)\end{array}$ & $\begin{array}{c}.002 \\
(.005)\end{array}$ & $\begin{array}{c}-.013 \\
(.005)\end{array}$ & $\begin{array}{c}-.033 \\
(.015)\end{array}$ & & $\begin{array}{r}-.006 \\
(.010)\end{array}$ & $\begin{array}{c}-.001 \\
(.005)\end{array}$ & $\begin{array}{c}.000 \\
(.004)\end{array}$ & & $\begin{array}{c}-.006 \\
(.004)\end{array}$ & $\begin{array}{c}-.002 \\
(.004)\end{array}$ & $\begin{array}{r}-.003 \\
(.005)\end{array}$ & $\begin{array}{r}-.006 \\
(.006)\end{array}$ & $\begin{array}{r}-.053 \\
(.013)\end{array}$ & & -.15 \\
\hline & & $\begin{array}{c}.001 \\
(.003)\end{array}$ & $\begin{array}{c}.000 \\
(.003)\end{array}$ & $\begin{array}{r}-.003 \\
(.003)\end{array}$ & $\begin{array}{c}.003 \\
(.003)\end{array}$ & $\begin{array}{r}-.002 \\
(.003)\end{array}$ & $\begin{array}{r}-.002 \\
(.004)\end{array}$ & $\begin{array}{r}-.006 \\
(.006)\end{array}$ & $\begin{array}{c}-.004 \\
(.005)\end{array}$ & $\begin{array}{c}.005 \\
(.008)\end{array}$ & $\begin{array}{c}.075 \\
(.016)\end{array}$ & $\begin{array}{c}-.001 \\
(.005)\end{array}$ & $\begin{array}{l}.005 \\
(.004)\end{array}$ & $\begin{array}{c}-.006 \\
(.004)\end{array}$ & $\begin{array}{c}-.002 \\
(.003)\end{array}$ & $\begin{array}{c}.001 \\
(.003)\end{array}$ & $\begin{array}{r}-.002 \\
(.004)\end{array}$ & $\begin{array}{r}-.003 \\
(.005)\end{array}$ & $\begin{array}{r}-.005 \\
(.005)\end{array}$ & $\begin{array}{c}-.021 \\
(.008)\end{array}$ & $\begin{array}{c}.136 \\
(.019)\end{array}$ \\
\hline & Experience $^{\mathrm{c}}$ & $\begin{array}{r}-.508 \\
(.139)\end{array}$ & $\begin{array}{r}-.150 \\
(.112)\end{array}$ & $\begin{array}{r}-.323 \\
(.117)\end{array}$ & $\begin{array}{c}-.050 \\
(.099)\end{array}$ & $\begin{array}{c}.006 \\
(.098)\end{array}$ & $\begin{array}{c}-.182 \\
(.118)\end{array}$ & $\begin{array}{c}-.118 \\
(.118)\end{array}$ & $\begin{array}{r}-.350 \\
(.127)\end{array}$ & $\begin{array}{c}-.209 \\
(.144)\end{array}$ & $\begin{array}{r}-.217 \\
(.133)\end{array}$ & $\begin{array}{c}-.313 \\
(.114)\end{array}$ & $\begin{array}{c}-.123 \\
(.096)\end{array}$ & $\begin{array}{c}-.153 \\
(.096)\end{array}$ & $\begin{array}{c}.074 \\
(.081)\end{array}$ & $\begin{array}{c}-.052 \\
(.075)\end{array}$ & $\begin{array}{r}-.111 \\
(.101)\end{array}$ & $\begin{array}{l}.070 \\
(.111)\end{array}$ & $\begin{array}{r}-.195 \\
(.105)\end{array}$ & $\begin{array}{c}-.283 \\
(.122)\end{array}$ & $\begin{array}{c}-.181 \\
(.129)\end{array}$ \\
\hline
\end{tabular}

a Covariances below diagonal and correlations above diagonal.

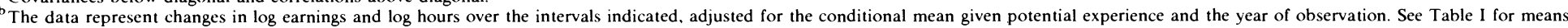

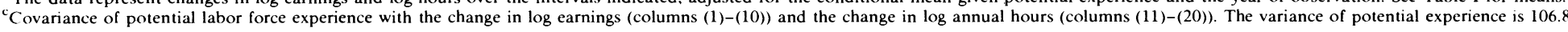




\section{TABLE V}

Covariances and Correlations Between Experience-Adjusted Changes in Earnings and Hours:

PSID MALES 1969 TO 1979, SEO EXCLUdED

(Standard Errors in Parentheses)

\begin{tabular}{|c|c|c|c|c|c|c|c|c|c|c|c|c|c|c|c|c|c|c|c|c|}
\hline with: & $\begin{array}{c}(1) \\
\Delta \log g \\
69-70\end{array}$ & $\begin{array}{c}(2) \\
\Delta \log g \\
70-71\end{array}$ & $\begin{array}{c}(3) \\
\Delta \log g \\
71-72\end{array}$ & $\begin{array}{c}(4) \\
\Delta \log g \\
72-73\end{array}$ & $\begin{array}{c}(5) \\
\Delta \log g \\
73-74\end{array}$ & $\begin{array}{c}(6) \\
\Delta \log g \\
74-75\end{array}$ & $\begin{array}{c}(7) \\
\Delta \log g \\
75-76\end{array}$ & $\begin{array}{c}(8) \\
\Delta \log g \\
76-77\end{array}$ & $\begin{array}{c}(9) \\
\Delta \log g \\
77-78\end{array}$ & $\begin{array}{c}\text { Covaria } \\
(10) \\
\Delta \log g \\
78-79\end{array}$ & $\begin{array}{c}\text { Ince of: } \\
(11) \\
\Delta \log h \\
69-70\end{array}$ & $\begin{array}{c}(12) \\
\Delta \log h \\
70-71\end{array}$ & $\begin{array}{c}(13) \\
\Delta \log h \\
71-72\end{array}$ & $\begin{array}{c}(14) \\
\Delta \log h \\
72-73\end{array}$ & $\begin{array}{c}(15) \\
\Delta \log h \\
73-74\end{array}$ & $\begin{array}{c}(16) \\
\Delta \log h \\
74-75\end{array}$ & $\begin{array}{c}(17) \\
\Delta \log h \\
75-76\end{array}$ & $\begin{array}{c}(18) \\
\Delta \log h \\
76-77\end{array}$ & $\begin{array}{c}(19) \\
\Delta \log h \\
77-78\end{array}$ & $\begin{array}{c}(20) \\
\Delta \log h \\
78-79\end{array}$ \\
\hline 1. $\begin{array}{c}\Delta \log g \\
69-70\end{array}$ & $\begin{array}{c}.146 \\
(.023)\end{array}$ & -.233 & -.005 & .002 & .020 & .018 & .041 & -.076 & .017 & -.023 & .556 & -.147 & .033 & .055 & -.013 & .076 & -.011 & -.023 & -.018 & -.019 \\
\hline 2. $\begin{array}{l}\Delta \log g \\
70-71\end{array}$ & $\begin{array}{r}-.033 \\
(.016)\end{array}$ & $\begin{array}{c}.138 \\
(.023)\end{array}$ & -.436 & -.152 & .034 & .012 & -.051 & -.029 & .057 & -.020 & -.081 & 0.569 & -.342 & -.015 & -.020 & 0.036 & -.085 & .023 & .022 & -.025 \\
\hline 3. $\begin{array}{l}\Delta \log g \\
71-72\end{array}$ & $\begin{array}{r}-.001 \\
(.009)\end{array}$ & $\begin{array}{r}-.060 \\
(.017)\end{array}$ & $\begin{array}{c}.137 \\
(.020)\end{array}$ & -.208 & -.049 & .004 & -.034 & .038 & -.002 & .008 & .054 & -.308 & .595 & -.087 & -.048 & -.039 & .035 & -.012 & .022 & -.025 \\
\hline 4. $\begin{array}{l}\Delta \log g \\
72-73\end{array}$ & $\begin{array}{c}.000 \\
(.004)\end{array}$ & $\begin{array}{r}-.018 \\
(.006)\end{array}$ & $\begin{array}{r}-.024 \\
(.007)\end{array}$ & $\begin{array}{c}.100 \\
(.013)\end{array}$ & -.341 & -.133 & .051 & -.018 & -.021 & -.046 & .030 & -.115 & .006 & .314 & -.013 & -.110 & -.005 & .033 & -.017 & .018 \\
\hline 5. $\begin{array}{l}\Delta \log g \\
73-74\end{array}$ & $\begin{array}{c}.002 \\
(.004)\end{array}$ & $\begin{array}{c}.004 \\
(.004)\end{array}$ & $\begin{array}{r}-.005 \\
(.005)\end{array}$ & $\begin{array}{r}-.032 \\
(.008)\end{array}$ & $\begin{array}{c}.089 \\
(.009)\end{array}$ & -.258 & -.007 & -.049 & -.002 & -.012 & .004 & .074 & -.095 & -.003 & .304 & -.100 & .021 & .012 & -.019 & -.058 \\
\hline 6. $\begin{array}{l}\Delta \log g \\
74-75\end{array}$ & $\begin{array}{c}.003 \\
(.004)\end{array}$ & $\begin{array}{c}.002 \\
(.005)\end{array}$ & $\begin{array}{c}.001 \\
(.005)\end{array}$ & $\begin{array}{r}-.015 \\
(.006)\end{array}$ & $\begin{array}{r}-.028 \\
(.007)\end{array}$ & $\begin{array}{c}.130 \\
(.018)\end{array}$ & -.342 & -.119 & -.065 & .060 & -.075 & .014 & .009 & -.020 & -.083 & .489 & -.228 & -.086 & -.053 & .039 \\
\hline 7. $\begin{array}{c}\Delta \log g \\
75-76\end{array}$ & $\begin{array}{c}.007 \\
(.005)\end{array}$ & $\begin{array}{r}-.009 \\
(.005)\end{array}$ & $\begin{array}{r}-.006 \\
(.005)\end{array}$ & $\begin{array}{c}.007 \\
(.007)\end{array}$ & $\begin{array}{r}-.001 \\
(.006)\end{array}$ & $\begin{array}{r}-.055 \\
(.016)\end{array}$ & $\begin{array}{c}.203 \\
(.042)\end{array}$ & -.537 & -.055 & .001 & .048 & -.057 & .019 & -.005 & -.006 & -.139 & 0.623 & -.342 & -.028 & -.059 \\
\hline 8. $\begin{array}{c}\Delta \log g \\
76-77\end{array}$ & $\begin{array}{r}-.012 \\
(.005)\end{array}$ & $\begin{array}{r}-.004 \\
(.005)\end{array}$ & $\begin{array}{c}.006 \\
(.005)\end{array}$ & $\begin{array}{r}-.002 \\
(.005)\end{array}$ & $\begin{array}{r}-.006 \\
(.004)\end{array}$ & $\begin{array}{r}-.017 \\
(.007)\end{array}$ & $\begin{array}{r}-.098 \\
(.038)\end{array}$ & $\begin{array}{c}.164 \\
(.041)\end{array}$ & -.157 & -.092 & -.055 & .008 & -.015 & .022 & .007 & -.131 & -.332 & .588 & -.064 & -.050 \\
\hline 9. $\begin{array}{c}\Delta \log g \\
77-78\end{array}$ & $\begin{array}{l}.003 \\
(.005)\end{array}$ & $\begin{array}{l}.008 \\
(.006)\end{array}$ & $\begin{array}{r}-.000 \\
(.004)\end{array}$ & $\begin{array}{r}-.003 \\
(.005)\end{array}$ & $\begin{array}{r}-.000 \\
(.004)\end{array}$ & $\begin{array}{r}-.009 \\
(.005)\end{array}$ & $\begin{array}{r}-.010 \\
(.010)\end{array}$ & $\begin{array}{r}-.024 \\
(.009)\end{array}$ & $\begin{array}{c}.149 \\
(.024)\end{array}$ & -.096 & .057 & .012 & .024 & -.070 & -.008 & -.025 & -.052 & -.109 & .557 & .060 \\
\hline
\end{tabular}




\begin{tabular}{|c|c|c|c|c|c|c|c|c|c|c|c|c|c|c|c|c|c|c|c|c|}
\hline 0. $\begin{array}{l}\Delta \log g \\
78-79\end{array}$ & $\begin{array}{c}-.003 \\
(.003)\end{array}$ & $\begin{array}{r}-.003 \\
(.005)\end{array}$ & $\begin{array}{c}.001 \\
(.005)\end{array}$ & $\begin{array}{r}-.006 \\
(.005)\end{array}$ & $\begin{array}{r}-.001 \\
(.004)\end{array}$ & $\begin{array}{c}.008 \\
(.004)\end{array}$ & $\begin{array}{c}.000 \\
(.005)\end{array}$ & $\begin{array}{r}-.014 \\
(.006)\end{array}$ & $\begin{array}{r}-.014 \\
(.012)\end{array}$ & $\begin{array}{l}.146 \\
(.020)\end{array}$ & .089 & .042 & .025 & -.030 & .057 & .005 & .015 & -.020 & .008 & .421 \\
\hline $\begin{array}{l}\Delta \log h \\
69-70\end{array}$ & $\begin{array}{c}.074 \\
(.019)\end{array}$ & $\begin{array}{r}-.010 \\
(.013)\end{array}$ & $\begin{array}{c}.007 \\
(.008)\end{array}$ & $\begin{array}{c}.003 \\
(.004)\end{array}$ & $\begin{array}{c}.000 \\
(.004)\end{array}$ & $\begin{array}{r}-.009 \\
(.005)\end{array}$ & $\begin{array}{c}.008 \\
(.006)\end{array}$ & $\begin{array}{r}-.008 \\
(.006)\end{array}$ & $\begin{array}{c}.008 \\
(.005)\end{array}$ & $\begin{array}{r}-.012 \\
(.006)\end{array}$ & $\begin{array}{l}.121 \\
(.020)\end{array}$ & -.317 & .005 & -.060 & .095 & -.045 & .014 & -.026 & .060 & -.091 \\
\hline $\begin{array}{l}\Delta \log h \\
70-71\end{array}$ & $\begin{array}{r}-.018 \\
(.013)\end{array}$ & $\begin{array}{c}.068 \\
(.020)\end{array}$ & $\begin{array}{r}-.037 \\
(.016)\end{array}$ & $\begin{array}{r}-.012 \\
(.004)\end{array}$ & $\begin{array}{c}.007 \\
(.003)\end{array}$ & $\begin{array}{c}.002 \\
(.003)\end{array}$ & $\begin{array}{r}-.008 \\
(.005)\end{array}$ & $\begin{array}{c}.001 \\
(.005)\end{array}$ & $\begin{array}{l}.002 \\
(.004)\end{array}$ & $\begin{array}{c}.005 \\
(.004)\end{array}$ & $\begin{array}{r}-.036 \\
(.013)\end{array}$ & $\begin{array}{c}.105 \\
(.020)\end{array}$ & -.526 & -.015 & -.026 & .042 & -.061 & .031 & -.056 & .037 \\
\hline $\begin{array}{l}\text { og } h \\
-72\end{array}$ & $\begin{array}{c}.004 \\
(.010)\end{array}$ & $\begin{array}{r}-.044 \\
(.018)\end{array}$ & $\begin{array}{c}.077 \\
(.019)\end{array}$ & $\begin{array}{c}.001 \\
(.006)\end{array}$ & $\begin{array}{r}-.010 \\
(.004)\end{array}$ & $\begin{array}{c}.001 \\
(.004)\end{array}$ & $\begin{array}{c}.003 \\
(.004)\end{array}$ & $\begin{array}{r}-.002 \\
(.004)\end{array}$ & $\begin{array}{c}.003 \\
(.004)\end{array}$ & $\begin{array}{c}.003 \\
(.004)\end{array}$ & $\begin{array}{c}.001 \\
(.011)\end{array}$ & $\begin{array}{r}-.060 \\
(.019)\end{array}$ & $\begin{array}{c}.122 \\
(.023)\end{array}$ & -.243 & -.109 & -.034 & .042 & -.022 & .031 & \\
\hline $\begin{array}{l}\text { og } h \\
-73\end{array}$ & $\begin{array}{l}.005 \\
(.004)\end{array}$ & $\begin{array}{r}-.001 \\
(.004)\end{array}$ & $\begin{array}{r}-.008 \\
(.005)\end{array}$ & $\begin{array}{c}.023 \\
(.005)\end{array}$ & $\begin{array}{r}-.000 \\
(.004)\end{array}$ & $\begin{array}{r}-.002 \\
(.003)\end{array}$ & $\begin{array}{r}-.001 \\
(.005)\end{array}$ & $\begin{array}{c}.002 \\
(.003)\end{array}$ & $\begin{array}{r}-.006 \\
(.003)\end{array}$ & $\begin{array}{r}-.003 \\
(.003)\end{array}$ & $\begin{array}{r}-.005 \\
(.004)\end{array}$ & $\begin{array}{r}-.00 \\
(.00\end{array}$ & $\begin{array}{r}-.020 \\
(.006)\end{array}$ & $\begin{array}{c}.054 \\
(.006)\end{array}$ & -.293 & -.011 & -.067 & .051 & -.065 & -.011 \\
\hline $\begin{array}{l}\Delta \log h \\
73-74\end{array}$ & $\begin{array}{r}-.001 \\
(.003)\end{array}$ & $\begin{array}{r}-.002 \\
(.004)\end{array}$ & $\begin{array}{r}-.004 \\
(.003)\end{array}$ & $\begin{array}{r}-.001 \\
(.005)\end{array}$ & $\begin{array}{c}.022 \\
(.006)\end{array}$ & $\begin{array}{r}-.007 \\
(.004)\end{array}$ & $\begin{array}{r}-.001 \\
(.004)\end{array}$ & $\begin{array}{c}.001 \\
(.004)\end{array}$ & $\begin{array}{r}-.001 \\
(.004)\end{array}$ & $\begin{array}{l}.005 \\
(.004)\end{array}$ & $\begin{array}{c}.008 \\
(.004)\end{array}$ & $\begin{array}{r}-.002 \\
(.003)\end{array}$ & $\begin{array}{r}-.009 \\
(.003)\end{array}$ & $\begin{array}{r}-.0 \\
(.0\end{array}$ & $\begin{array}{c}.057 \\
(.006)\end{array}$ & -.381 & .016 & .000 & .025 & -.061 \\
\hline $\begin{array}{l}\operatorname{og} h \\
-75\end{array}$ & $\begin{array}{c}.008 \\
(.004)\end{array}$ & $\begin{array}{c}.004 \\
(.004)\end{array}$ & $\begin{array}{r}-.004 \\
(.004)\end{array}$ & $\begin{array}{r}-.010 \\
(.004)\end{array}$ & $\begin{array}{r}-.009 \\
(.005)\end{array}$ & $\begin{array}{c}.051 \\
(.012)\end{array}$ & $\begin{array}{r}-.018 \\
(.012)\end{array}$ & $\begin{array}{r}-.015 \\
(.008)\end{array}$ & $\begin{array}{r}-.003 \\
(.004)\end{array}$ & $\begin{array}{c}.001 \\
(.004)\end{array}$ & $\begin{array}{r}-.004 \\
(.005)\end{array}$ & $\begin{array}{c}.004 \\
(.004)\end{array}$ & $\begin{array}{r}-.003 \\
(.005)\end{array}$ & $\begin{array}{r}-.001 \\
(.003)\end{array}$ & $\begin{array}{r}-.026 \\
(.005)\end{array}$ & $\begin{array}{c}.083 \\
(.012)\end{array}$ & -.348 & -.150 & -.042 & \\
\hline $\begin{array}{c}\Delta \log h \\
75-76\end{array}$ & $\begin{array}{r}-.001 \\
(.004)\end{array}$ & $\begin{array}{r}-.011 \\
(.005)\end{array}$ & $\begin{array}{c}.004 \\
(.004)\end{array}$ & $\begin{array}{r}-.001 \\
(.004)\end{array}$ & $\begin{array}{c}.002 \\
(.004)\end{array}$ & $\begin{array}{r}-.028 \\
(.011)\end{array}$ & $\begin{array}{c}.096 \\
(.027)\end{array}$ & $\begin{array}{r}-.046 \\
(.027)\end{array}$ & $\begin{array}{r}-.007 \\
(.005)\end{array}$ & $\begin{array}{l}.002 \\
(.003)\end{array}$ & $\begin{array}{l}.002 \\
(.004)\end{array}$ & $\begin{array}{r}-.007 \\
(.004)\end{array}$ & $\begin{array}{c}.005 \\
(.004)\end{array}$ & $\begin{array}{r}-.005 \\
(.003)\end{array}$ & $\begin{array}{c}.001 \\
(.003)\end{array}$ & $\begin{array}{r}-.034 \\
(.011)\end{array}$ & $\begin{array}{c}.118 \\
(.023)\end{array}$ & -.528 & -.048 & -.016 \\
\hline $\begin{array}{l}\Delta \log h \\
76-77\end{array}$ & $\begin{array}{r}-.003 \\
(.005)\end{array}$ & $\begin{array}{c}.003 \\
(.005)\end{array}$ & $\begin{array}{r}-.002 \\
(.004)\end{array}$ & $\begin{array}{c}.004 \\
(.004)\end{array}$ & $\begin{array}{c}.001 \\
(.004)\end{array}$ & $\begin{array}{r}-.011 \\
(.006)\end{array}$ & $\begin{array}{r}-.054 \\
(.031)\end{array}$ & $\begin{array}{c}.083 \\
(.034)\end{array}$ & $\begin{array}{r}-.015 \\
(.008)\end{array}$ & $\begin{array}{r}-.003 \\
(.004)\end{array}$ & $\begin{array}{r}-.003 \\
(.006)\end{array}$ & $\begin{array}{c}.003 \\
(.004)\end{array}$ & $\begin{array}{r}-.003 \\
(.004)\end{array}$ & $\begin{array}{c}.004 \\
(.002)\end{array}$ & $\begin{array}{c}.000 \\
(.004)\end{array}$ & $\begin{array}{r}-.015 \\
(.007)\end{array}$ & $\begin{array}{r}-.064 \\
(.024)\end{array}$ & $\begin{array}{c}.123 \\
(.030)\end{array}$ & -.217 & -.070 \\
\hline $\begin{array}{l}\Delta \log h \\
77-78\end{array}$ & $\begin{array}{r}-.003 \\
(.005)\end{array}$ & $\begin{array}{c}.003 \\
(.005)\end{array}$ & $\begin{array}{c}.003 \\
(.004)\end{array}$ & $\begin{array}{r}-.002 \\
(.003)\end{array}$ & $\begin{array}{r}-.002 \\
(.004)\end{array}$ & $\begin{array}{r}-.007 \\
(.004)\end{array}$ & $\begin{array}{r}-.005 \\
(.005)\end{array}$ & $\begin{array}{r}-.010 \\
(.006)\end{array}$ & $\begin{array}{c}.080 \\
(.020)\end{array}$ & $\begin{array}{c}.001 \\
(.009)\end{array}$ & $\begin{array}{c}.008 \\
(.007)\end{array}$ & $\begin{array}{r}-.007 \\
(.005)\end{array}$ & $\begin{array}{c}.004 \\
(.005)\end{array}$ & $\begin{array}{r}-.006 \\
(.003)\end{array}$ & $\begin{array}{l}.00 \\
(.00\end{array}$ & $\begin{array}{r}-.005 \\
(.004)\end{array}$ & $\begin{array}{r}-.006 \\
(.005)\end{array}$ & $\begin{array}{r}-.028 \\
(.008)\end{array}$ & $\begin{array}{c}.139 \\
(.026)\end{array}$ & -.245 \\
\hline $\begin{array}{l}\Delta \log h \\
78-79\end{array}$ & $\begin{array}{r}-.003 \\
(.004)\end{array}$ & $\begin{array}{r}-.004 \\
(.004)\end{array}$ & $\begin{array}{c}.004 \\
(.003)\end{array}$ & $\begin{array}{c}.002 \\
(.005)\end{array}$ & $\begin{array}{r}-.007 \\
(.005)\end{array}$ & $\begin{array}{c}.006 \\
(.004)\end{array}$ & $\begin{array}{r}-.011 \\
(.007)\end{array}$ & $\begin{array}{r}-.008 \\
(.006)\end{array}$ & $\begin{array}{c}.009 \\
(.010)\end{array}$ & $\begin{array}{c}.065 \\
(.015)\end{array}$ & $\begin{array}{r}-.013 \\
(.006)\end{array}$ & $\begin{array}{c}.005 \\
(.003)\end{array}$ & $\begin{array}{l}.002 \\
(.004)\end{array}$ & $\begin{array}{r}-.001 \\
(.003)\end{array}$ & $\begin{array}{r}-.006 \\
(.005)\end{array}$ & $\begin{array}{c}.005 \\
(.004)\end{array}$ & $\begin{array}{r}-.002 \\
(.004)\end{array}$ & $\begin{array}{r}-.010 \\
(.006)\end{array}$ & $\begin{array}{r}-.037 \\
(.017)\end{array}$ & $\begin{array}{l}.102 \\
(.030)\end{array}$ \\
\hline Experience $^{\mathrm{C}}$ & $\begin{array}{r}-.717 \\
(.172)\end{array}$ & $\begin{array}{r}-.300 \\
(.145)\end{array}$ & $\begin{array}{r}-.320 \\
(.143)\end{array}$ & $\begin{array}{r}-.060 \\
(.110)\end{array}$ & $\begin{array}{r}-.276 \\
(.105)\end{array}$ & $\begin{array}{r}-.234 \\
(.124)\end{array}$ & $\begin{array}{r}-.118 \\
(.130)\end{array}$ & $\begin{array}{r}-.154 \\
(.123)\end{array}$ & $\begin{array}{r}-.552 \\
(.159)\end{array}$ & $\begin{array}{r}-.260 \\
(.151)\end{array}$ & $\begin{array}{r}-.319 \\
(.150)\end{array}$ & $\begin{array}{r}-.277 \\
(.129)\end{array}$ & $\begin{array}{r}-.092 \\
(.137)\end{array}$ & $\begin{array}{r}-.011 \\
(.086)\end{array}$ & $\begin{array}{r}-.092 \\
(.085)\end{array}$ & $\begin{array}{r}-.276 \\
(.111)\end{array}$ & $\begin{array}{c}.122 \\
(.108)\end{array}$ & $\begin{array}{r}-.204 \\
(.112)\end{array}$ & $\begin{array}{r}-.241 \\
(.154)\end{array}$ & $\begin{array}{r}-.289 \\
(.151)\end{array}$ \\
\hline
\end{tabular}

${ }^{a}$ Covariances below diagonal and correlations above diagonal

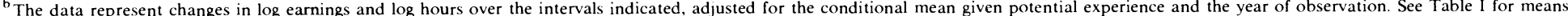

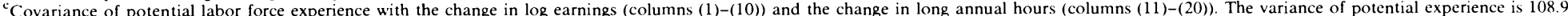


TABLE VI

COVARIANCES AND CORRELATIONS ${ }^{\mathrm{a}}$ of EXPERIENCE-AdJusted ChANGES IN EARNINGS AND HOURS: NLS DATA $1966-75^{\mathrm{b}}$

(Standard Errors in Parentheses)

\begin{tabular}{|c|c|c|c|c|c|c|c|c|c|c|}
\hline with: & $\begin{array}{c}(1) \\
\Delta \log g \\
66-67\end{array}$ & $\begin{array}{c}(2) \\
\Delta \log g \\
67-69\end{array}$ & $\begin{array}{c}(3) \\
\Delta \log g \\
69-71\end{array}$ & $\begin{array}{c}(4) \\
\Delta \log g \\
71-73\end{array}$ & $\begin{array}{l}\text { Covarianc } \\
\quad(5) \\
\Delta \log g \\
73-75\end{array}$ & $\begin{array}{l}\text { ce of: } \\
\quad \text { (6) } \\
\Delta \log h \\
66-67\end{array}$ & $\begin{array}{c}(7) \\
\Delta \log h \\
67-69\end{array}$ & $\begin{array}{c}(8) \\
\Delta \log h \\
69-71\end{array}$ & $\begin{array}{c}(9) \\
\Delta \log h \\
71-73\end{array}$ & $\begin{array}{c}(10) \\
\Delta \log h \\
73-75\end{array}$ \\
\hline 1. $\begin{array}{c}\Delta \log g \\
66-67\end{array}$ & $\begin{array}{c}.109 \\
(.016)\end{array}$ & -.27 & .00 & -.04 & .02 & .34 & -.07 & .02 & .01 & .01 \\
\hline 2. $\begin{array}{c}\Delta \log g \\
67-69\end{array}$ & $\begin{array}{r}-.032 \\
(.010)\end{array}$ & $\begin{array}{l}.133 \\
(.039)\end{array}$ & -.51 & .00 & .03 & .02 & .15 & -.07 & -.01 & .05 \\
\hline 3. $\begin{array}{l}\Delta \log g \\
69-71\end{array}$ & $\begin{array}{r}-.000 \\
(.004)\end{array}$ & $\begin{array}{r}-.068 \\
(.038)\end{array}$ & $\begin{array}{c}.132 \\
(.040)\end{array}$ & -.26 & -.05 & .02 & -.04 & 16 & -.06 & -.03 \\
\hline 4. $\begin{array}{l}\Delta \log g \\
71-73\end{array}$ & $\begin{array}{r}-.005 \\
(.004)\end{array}$ & $\begin{array}{l}.000 \\
(.004)\end{array}$ & $\begin{array}{r}-.040 \\
(.008)\end{array}$ & $\begin{array}{l}.178 \\
(.024)\end{array}$ & -.30 & -.02 & -.01 & -.02 & .47 & -.17 \\
\hline 5. $\begin{array}{c}\Delta \log g \\
73-75\end{array}$ & $\begin{array}{c}.004 \\
(.006)\end{array}$ & $\begin{array}{c}.007 \\
(.010)\end{array}$ & $\begin{array}{c}-.011 \\
(.010)\end{array}$ & $\begin{array}{r}-.073 \\
(.018)\end{array}$ & $\begin{array}{l}.330 \\
(.036)\end{array}$ & .03 & -.10 & .09 & -.15 & .71 \\
\hline 6. $\begin{array}{l}\Delta \log h \\
66-67\end{array}$ & $\begin{array}{c}.038 \\
(.012)\end{array}$ & $\begin{array}{l}.002 \\
(.005)\end{array}$ & $\begin{array}{c}.002 \\
(.003)\end{array}$ & $\begin{array}{c}-.003 \\
(.004)\end{array}$ & $\begin{array}{c}.005 \\
(.006)\end{array}$ & $\begin{array}{c}.114 \\
(.023)\end{array}$ & -.37 & -.02 & -.01 & .03 \\
\hline 7. $\Delta \log h$ & $\begin{array}{r}-.007 \\
(.004)\end{array}$ & $\begin{array}{c}.016 \\
(.004)\end{array}$ & $\begin{array}{r}-.004 \\
(.004)\end{array}$ & $\begin{array}{c}.001 \\
(.005)\end{array}$ & $\begin{array}{r}-.017 \\
(.007)\end{array}$ & $\begin{array}{r}-.036 \\
(.012)\end{array}$ & $\begin{array}{c}.081 \\
(.015)\end{array}$ & -.52 & .08 & -.09 \\
\hline 8. $\Delta \log h$ & $\begin{array}{l}.002 \\
(.003)\end{array}$ & $\begin{array}{r}-.007 \\
(.004)\end{array}$ & $\begin{array}{l}.015 \\
(.004)\end{array}$ & $\begin{array}{l}.002 \\
(.006)\end{array}$ & $\begin{array}{l}.013 \\
(.005)\end{array}$ & $\begin{array}{r}-.002 \\
(.003)\end{array}$ & $\begin{array}{r}-.038 \\
(.008)\end{array}$ & $\begin{array}{c}.066 \\
(.011)\end{array}$ & -.36 & -.09 \\
\hline 9. $\begin{array}{l}\Delta \log h \\
71-73\end{array}$ & $\begin{array}{l}.001 \\
(.003)\end{array}$ & $\begin{array}{c}-.001 \\
(.004)\end{array}$ & $\begin{array}{r}-.007 \\
(.005)\end{array}$ & $\begin{array}{c}.060 \\
(.014)\end{array}$ & $\begin{array}{c}-.026 \\
(.009)\end{array}$ & $\begin{array}{l}-.001 \\
(.003)\end{array}$ & $\begin{array}{l}.007 \\
(.005)\end{array}$ & $\begin{array}{r}-.028 \\
(.007)\end{array}$ & $\begin{array}{c}.091 \\
(.014)\end{array}$ & -.34 \\
\hline 10. $\begin{array}{l}\Delta \log h \\
73-75\end{array}$ & $\begin{array}{l}.002 \\
(.007)\end{array}$ & $\begin{array}{c}.009 \\
(.009)\end{array}$ & $\begin{array}{r}-.006 \\
(.009)\end{array}$ & $\begin{array}{r}-.034 \\
(.010)\end{array}$ & $\begin{array}{l}.198 \\
(.027)\end{array}$ & $\begin{array}{l}.005 \\
(.005)\end{array}$ & $\begin{array}{r}-.013 \\
(.006)\end{array}$ & $\begin{array}{l}.011 \\
(.005)\end{array}$ & $\begin{array}{r}-.050 \\
(.010)\end{array}$ & $\begin{array}{l}.235 \\
(.028)\end{array}$ \\
\hline 11. Experience ${ }^{\mathfrak{c}}$ & $\begin{array}{l}.062 \\
(.051)\end{array}$ & $\begin{array}{c}.078 \\
(.063)\end{array}$ & $\begin{array}{c}.016 \\
(.057)\end{array}$ & $\begin{array}{r}-.160 \\
(.056)\end{array}$ & $\begin{array}{l}-.225 \\
(.086)\end{array}$ & $\begin{array}{r}-.018 \\
(.049)\end{array}$ & $\begin{array}{l}.000 \\
(.048)\end{array}$ & $\begin{array}{c}.052 \\
(.036)\end{array}$ & $\begin{array}{r}-.158 \\
(.041)\end{array}$ & $\begin{array}{c}-.291 \\
(.078)\end{array}$ \\
\hline
\end{tabular}

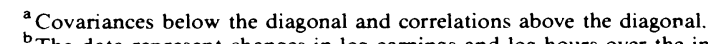

b The data represent changes in log earnings and log hours over the intervals indicated, adjusted for the conditional mean given potential experience and the year of observation. See Table II for means.

${ }^{c}$ Covariance of potential labor market experience with the change in log earnings (columns (1)-(5)) and the change in log hours (columns $(6)-(10)$ ). The variance of potential experience is 26.6.

Table VI presents the covariance matrix of experience-adjusted changes in earnings and hours from our NLS sample. Overall, the data are very similar to the corresponding data from the PSID, although there is more evidence of nonstationarity in the NLS data. On the other hand, none of the second-order autocovariances or cross-covariances in Table VI are large or statistically significant, so that the NLS data may perhaps be adequately summarized as a nonstationary bivariate first-order moving average process. Assuming that changes in annual earnings and hours between consecutive years are generated by a second-order moving average process, however, changes in earnings and hours at two-year gaps can be represented by a first-order moving average. ${ }^{11}$ Thus the

\footnotetext{
${ }^{11}$ If the change in earnings $\left(\Delta \log g_{t}\right)$ is $\operatorname{MA}(2)$, then $\Delta \log g_{t}=\varepsilon_{t}+b_{1} \varepsilon_{t-1}+b_{2} \varepsilon_{t-2}$, for example, where $\varepsilon_{t}$ is serially uncorrelated. The change in earnings between period $t-2$ and period $t$ is $\log g_{t}-\log g_{t-2}=\Delta \log g_{t}+\Delta \log g_{t-1}$. Therefore, $\log g_{t}-\log g_{t-2}=\varepsilon_{t}+\left(1+b_{1}\right) \varepsilon_{t-1}+\left(b_{1}+\right.$ $\left.b_{2}\right) \varepsilon_{t-2}+b_{2} \varepsilon_{t-3}$. Notice that the change over two years is correlated with its first lag, but not with its second.
} 
data in Table VI are potentially consistent with the same underlying model of earnings and hours generation as in Tables IV and V.

Table VII presents the covariances of experience-adjusted changes in earnings and hours between consecutive six-month intervals in the SIME/DIME survey. Again, these covariances are similar to the covariances of the PSID and NLS samples. There is less evidence of nonstationarity in the SIME/DIME sample than in the other samples, perhaps as a result of the averaging of earnings and hours in the SIME/DIME survey over several different calendar periods. The first-order autocorrelations of earnings and hours changes are similar between the SIME/DIME sample and the other samples. The covariances between contemporaneous changes in earnings and hours, on the other hand, are relatively higher in the SIME/DIME sample. This implies that the simple regression coefficient of changes in the logarithm of annual hours on changes in the logarithm of average hourly earnings is more positive in the SIME/DIME sample $(-.17)$ than in the other surveys $(-.31$ in the overall PSID sample, -.36 in the PSID sample without the SEO, and -.32 in the NLS). None of the third-order autocovariances or cross-covariances is statistically significant in Table VII, suggesting that semi-annual changes in log earnings and log hours in the SIME/DIME sample are close to a bivariate $\mathrm{MA}(2)$ process. Since a bivariate MA(2) representation of semi-annual changes in earnings and hours implies only a first-order moving average representation of annual changes, the SIME/DIME data therefore exhibit lower order serial correlation than either the PSID or NLS data.

The covariances of changes in earnings and hours with potential labor market experience are presented for each sample in the bottom rows of Tables IV-VII. These covariances vary from year-to-year within each sample and also vary in relative magnitude across the samples. In the two PSID samples the average covariance of experience with the change in earnings is about 1.7 times larger than the corresponding covariance with the change in hours. In the NLS sample, by comparison, the average covariance of experience with the change in earnings is about one-third as large as the covariance with the change in hours. Finally, for the SIME/DIME sample the ratio of the average covariances of earnings and hours changes with potential experience is 2.8 . These differences across samples suggest that the relative curvature of the lifecycle profiles of earnings and hours vary considerably with the age and demographic composition of the sample. ${ }^{12}$

We next consider whether the information in the covariance matrices in Tables IV-VII may be adequately summarized by some relatively simple statistical model. Table VIII presents several tests that address this concern. Row 1a presents the $\chi^{2}$ goodness-of-fit statistic for a nonstationary bivariate MA(2) representation of earnings and hours changes from each survey. ${ }^{13}$ The goodness-

\footnotetext{
${ }^{12}$ As noted by Ghez and Becker (1975), the relative curvature of the life-cycle profiles of earnings and hours provides a means of estimating the intertemporal substitution elasticity. See our earlier paper (Abowd and Card (1986)) for further details in the present context.

${ }^{13}$ The timing interval of the underlying bivariate $\mathrm{MA}(2)$ model is assumed to correspond to the timing interval of each survey, i.e., to changes in annual data for the PSID; to changes in annual data over a two-year gap for the NLS; and to changes in semi-annual data for the SIME/DIME data.
} 


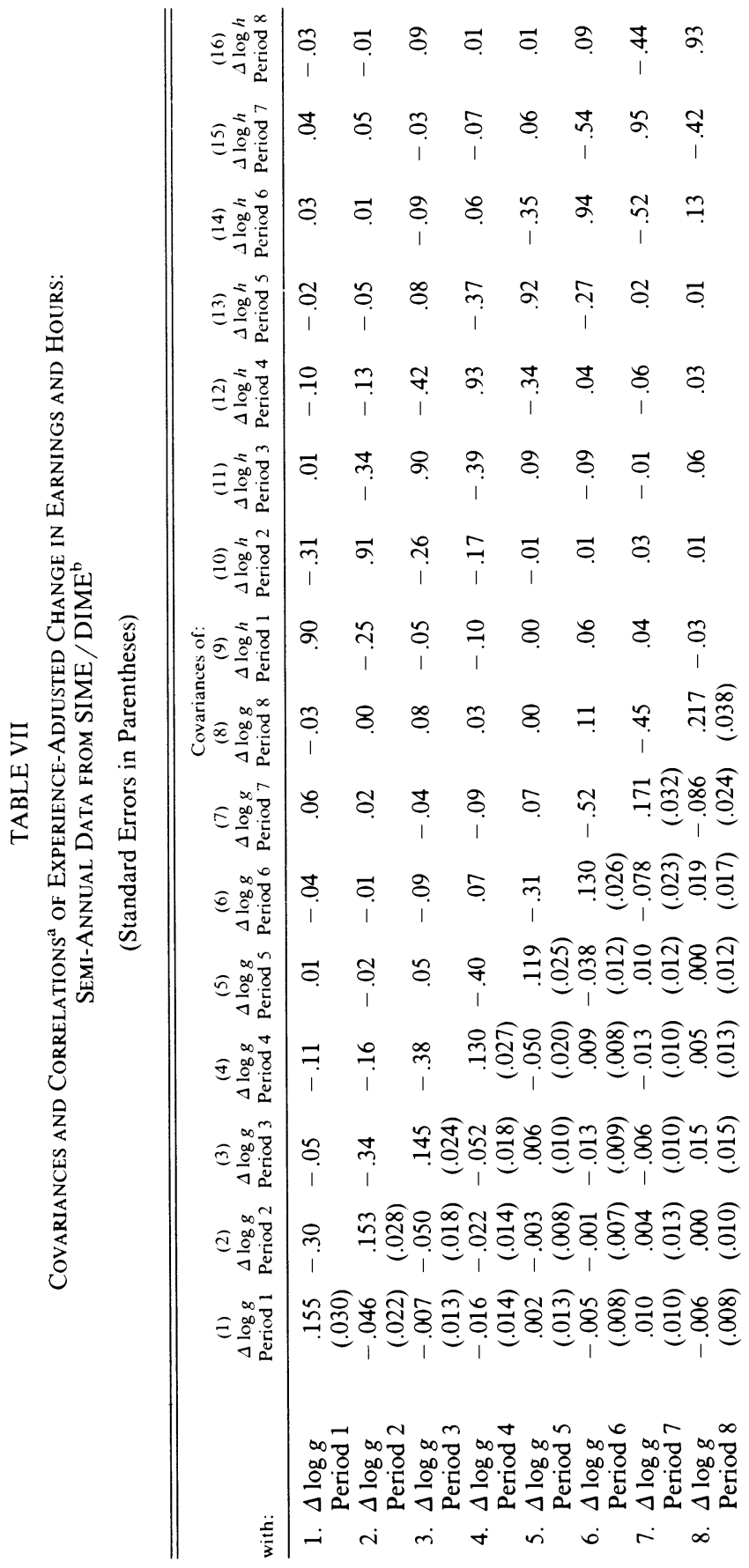




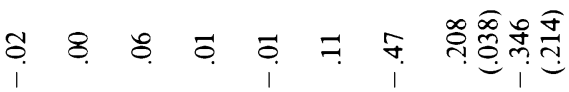

ㄷ. \& o

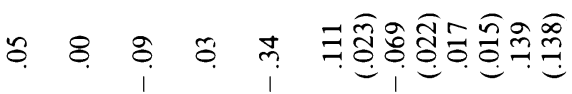

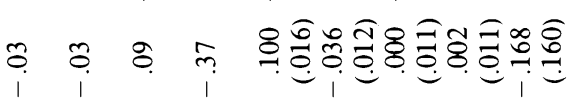

o

ㄱ

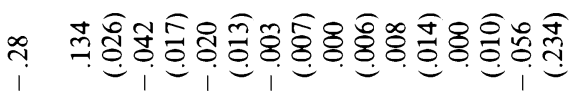

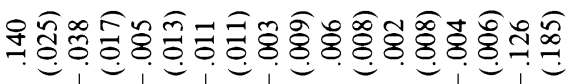

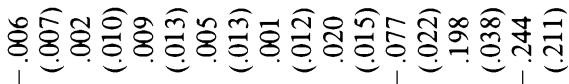

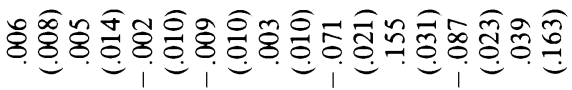

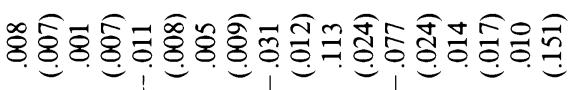

\&

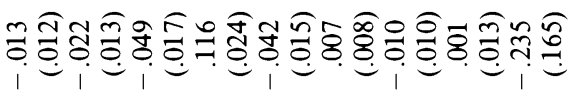

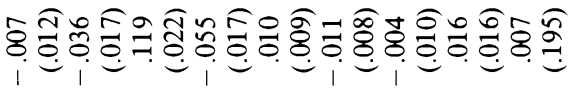

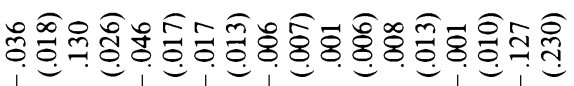

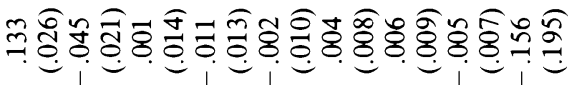

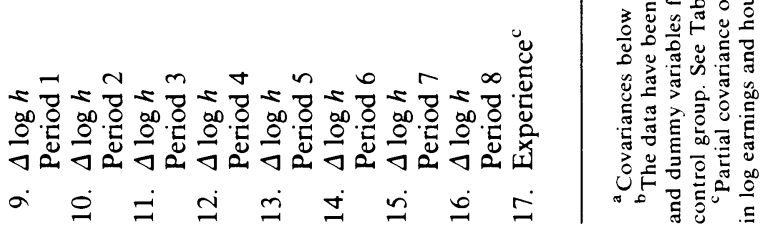


of-fit statistic is formed according to the formulas presented in Appendix A, using the actual sampling variability of the second moments of the data (rather than assuming, for example, that the underlying earnings and hours data follow a multivariate normal distribution). For all four samples the test statistics confirm that the autocovariances and cross-covariances at lags greater than two periods are negligible. Row $1 \mathrm{~b}$ shows the importance of recognizing that earnings and hours data are not normally distributed. In this row the same statistics are computed using the formulas for the sampling variability of the second moments of earnings and hours changes implied by multivariate normality. ${ }^{14}$ Evidently, tests based on the assumption of joint normality can be misleading, and in the present context would lead to rejection of the bivariate MA(2) hypothesis. Based on the test statistics in Row 1a, however, we accept the restrictions implied by the bivariate MA(2) hypothesis.

Having accepted this model, it is of some interest to compare our results to previous studies of the covariance structure of earnings. ${ }^{15}$ It is also interesting to consider which models of earnings and hours are ruled out by the MA(2) model. The most directly comparable previous study is MaCurdy's (1982) analysis of percentage changes in annual earnings for data from the first 10 waves of the PSID. In his study, MaCurdy finds that changes in earnings are adequately summarized by a second-order moving average. His estimates of the average autocorrelations of earnings changes (MaCurdy (1982, p. 107)) are very similar to estimates of the average autocorrelations of earnings changes from our PSID samples. In contrast to our finding of significant nonstationarity, however, MaCurdy accepts a stationary MA(2) representation of the change in earnings. We suspect that the difference is due to alternative treatments of outliers. In our analysis we have excluded only those individuals in the PSID sample who report average hourly earnings in excess of $\$ 100$, or annual hours in excess of 4680 . MaCurdy (1982) adopts a more stringent set of exclusion restrictions. Nonetheless, our conclusions from the PSID sample are broadly consistent with MaCurdy's.

Other studies of the longitudinal earnings data include Lillard and Weiss (1979) and Hause (1980). Lillard and Weiss (1979) analyze the level of log earnings for research scientists from the National Science Foundation's Register of Technical and Scientific Personnel. Their sample contains annual earnings information for alternating years (the structure of our NLS sample). From their reported covariance matrix (Lillard and Weiss $(1979$, p. 443)) we can calculate

${ }^{14}$ If the data are normally distributed, then estimates of the sampling variability of the second moments can be obtained from the second moments themselves: See Anderson (1958, p. 75). In particular, if a vector is normally distributed with variance matrix $\Sigma$, then the sampling covariance of $\sigma_{i j}$, the $(i, j)$ element of $\Sigma$, with $\sigma_{u v}$, the $(u, v)$ element of $\Sigma$, can be estimated by

$$
\hat{\sigma}_{i u} \hat{\sigma}_{j v}+\hat{\sigma}_{i n} \hat{\sigma}_{j u} \text {, }
$$

where $\hat{\sigma}_{i j}$ is the maximum likelihood estimate of $\boldsymbol{\sigma}_{i j}$.

${ }_{15}$ We were unable to find any previously published studies of the autocovariance structure of hours from which we could recover an autocovariance matrix. 
the implied covariance matrix for the changes in log earnings; we cannot, however, calculate a goodness-of-fit statistic for the second order moving average model. Nevertheless, it is instructive to note that the first order autocorrelations of changes in earnings in Lillard and Weiss' data are negative (between -.14 and -.46 ). The second order autocorrelations are smaller, but all positive (between .07 and .09). There is also substantial evidence of nonstationarity in Lillard and Weiss' estimates of the variances of earnings changes. Finally, one of the higher-order autocovariances of earnings changes in Lillard and Weiss' sample is relatively large, suggesting that an MA(2) representation of their data is inadequate.

Hause (1980) presents annual earnings data for a sample of young Swedish males. His reported covariance matrix of the levels of log earnings (1980, p. 1025) implies a covariance matrix for the changes in log earnings that exhibits negative first order autocorrelations ranging from -.17 to -.23 -somewhat smaller in absolute value than the comparable correlations of earnings in Tables IV-VII. The second order autocorrelations are also negative $(-.05$ to -.20$)$ but the third and fourth order autocorrelations are generally close to zero. The implied variances of the changes in log earnings in Hause's data also display considerable nonstationarity.

Taken as a whole, the evidence on changes in earnings from the samples in this paper and the two additional samples considered by Lillard and Weiss and Hause suggests that negative serial correlation between consecutive changes in log earnings is a pervasive phenomenon. Nonstationarity of the covariances also appears to be an important property of longitudinal data. Finally, although the samples used in this paper show little evidence of higher order autocorrelations among earnings changes, at least one previous study (Lillard and Weiss) offers some contrary evidence.

An important implication of the bivariate MA(2) structure of earnings and hours changes is the absence of any permanent individual components of variance in the rate of growth of earnings or hours. Specifically, the random trend models of Lillard and Weiss (1979) and Hause (1980) are inconsistent with the data in Tables IV-VI. ${ }^{16}$ Individual-specific trends in the growth rates of earnings and hours imply that experience-adjusted changes in earnings and hours $\left(\Delta \log \tilde{g}_{i t}\right.$ and $\Delta \log \tilde{h}_{i t}$, respectively) satisfy

$$
\begin{aligned}
& \Delta \log \tilde{g}_{i t}=\phi_{1 i}+\varepsilon_{1 i t}, \\
& \Delta \log \tilde{h}_{i t}=\phi_{2 i}+\varepsilon_{2 i t},
\end{aligned}
$$

where $\phi_{1 i}$ and $\phi_{2 i}$ are the individual-specific growth rates of earnings and hours (expressed as deviations from population growth rates), and $e_{1 i t}$ and $\varepsilon_{2 i t}$ represent the deviations of growth rates from their individual-specific means. These

\footnotetext{
${ }^{16} \mathrm{MaCurdy}$ (1982) reaches a similar conclusion with respect to the presence of random earnings trends.
} 
equations imply

$$
\begin{aligned}
& \operatorname{cov}\left[\Delta \log \tilde{g}_{i t}, \Delta \log \tilde{g}_{i s}\right]=\operatorname{var}\left[\phi_{1 i}\right]+\operatorname{cov}\left[\varepsilon_{1 i t}, \varepsilon_{1 i s}\right], \\
& \operatorname{cov}\left[\Delta \log \tilde{h}_{i t}, \Delta \log \tilde{h}_{i s}\right]=\operatorname{var}\left[\phi_{2 i}\right]+\operatorname{cov}\left[\varepsilon_{2 i t}, \varepsilon_{2 i s}\right], \\
& \operatorname{cov}\left[\Delta \log \tilde{g}_{i t}, \Delta \log \tilde{h}_{i s}\right]=\operatorname{cov}\left[\phi_{1 i}, \phi_{2 i}\right]+\operatorname{cov}\left[\varepsilon_{1 i t}, \varepsilon_{2 i s}\right] .
\end{aligned}
$$

According to these equations, the autocovariances of earnings and hours changes contain fixed positive components associated with the cross-sectional variation in individual-specific growth rates. The evidence that the higher-order autocovariances of earnings and hours changes are jointly equal to zero, however, suggests $\operatorname{var}\left[\phi_{1 i}\right]=0$ and $\operatorname{var}\left[\phi_{2 i}\right]=0$.

A second important implication of the bivariate $\mathrm{MA}(2)$ representation of earnings and hours changes is that any autoregressive error components of the levels of individual earnings and hours have (approximately) unit roots. ${ }^{17}$ The only autoregressive error process for the levels of earnings or hours that is consistent with the bivariate MA(2) model for the changes is one with a unit autoregressive coefficient. ${ }^{18}$

A final implication of the bivariate $\mathrm{MA}(2)$ representation is that individualspecific responses to aggregate business cycle movements are negligible components of variance in earnings and hours changes. Specifically, consider a model in which earnings and hours respond to changes in some aggregate business-cycle indicator $\left(\Delta y_{t}\right)$ with individual-specific elasticities $\beta_{1 i}$ and $\beta_{2 i}$, respectively:

$$
\begin{aligned}
& \Delta \log \tilde{g}_{i t}=\beta_{1 i} \Delta y_{t}+\varepsilon_{1 i t}, \\
& \Delta \log \tilde{h}_{i t}=\beta_{2 i} \Delta y_{t}+\varepsilon_{2 i t} .
\end{aligned}
$$

In these equations $\varepsilon_{1 i t}$ and $\varepsilon_{2 i t}$ represent cyclically adjusted changes in individual earnings and hours (controlling for experience). This model implies

$$
\begin{aligned}
& \operatorname{cov}\left[\Delta \log \tilde{g}_{i t}, \Delta \log \tilde{g}_{i s}\right]=\operatorname{var}\left[\beta_{1 i}\right] \Delta y_{t} \Delta y_{s}+\operatorname{cov}\left[\varepsilon_{1 i t}, \varepsilon_{1 i s}\right], \\
& \operatorname{cov}\left[\Delta \log \tilde{h}_{i t}, \Delta \log \tilde{h}_{i s}\right]=\operatorname{var}\left[\beta_{2 i}\right] \Delta y_{t} \Delta y_{s}+\operatorname{cov}\left[\varepsilon_{2 i t}, \varepsilon_{2 i s}\right], \\
& \operatorname{cov}\left[\Delta \log \tilde{g}_{i t}, \Delta \log \tilde{h}_{i s}\right]=\operatorname{cov}\left[\beta_{1 i}, \beta_{2 i}\right] \Delta y_{t} \Delta y_{s}+\operatorname{cov}\left[\varepsilon_{1 i t}, \varepsilon_{2 i s}\right] .
\end{aligned}
$$

According to these equations, the covariances between changes in earnings or hours in one year and change in any other year depend on the signs and magnitudes of the business cycle movements in those years. For example, covariances between 1969-70 changes and 1974-75 changes in our PSID samples should be substantially different from covariances between 1970-71 changes and 1975-76 changes, because business-cycle conditions moved in the same direction between 1969-70 and 1974-75 $\left(\Delta y_{t} \Delta y_{s}>0\right)$ and in the opposite direction between 1970-71 and 1975-76 $\left(\Delta y_{t} \Delta y_{s}<0\right)$. The data in Tables IV and V,

\footnotetext{
${ }^{17}$ MaCurdy (1982) also concludes that there are not stationary autoregressive components of variance in the PSID earnings data.

${ }^{18}$ Since the focus of this paper is on statistical models for the changes in earnings and hours, we have not directly examined the covariance matrices of experience-adjusted levels of earnings and hours.
} 
however, show that the third and higher-order covariances of earnings and hours changes are all approximately zero, regardless of the cyclical changes associated with particular years. On this basis we conclude that cross-sectional variation in the cyclical responsiveness of earnings and hours is negligible (i.e., $\operatorname{var}\left[\beta_{1 i}\right]=0$, $\left.\operatorname{var}\left[\beta_{2 i}\right]=0, \operatorname{cov}\left[\beta_{1 i}, \beta_{2 i}\right]=0\right)$.

Rows 2 and 3 of Table VIII test further simplifications of the covariance structure against the alternative of a nonstationary bivariate $\operatorname{MA}(2)$. The $\chi^{2}$ statistics in Row 2 test whether a stationary MA(2) representation is adequate. ${ }^{19}$ All samples show strong evidence of nonstationarity. The $\chi^{2}$ statistics in Row 3 test whether a nonstationary $\mathrm{MA}(1)$ representation is adequate (i.e., whether the second order autocovariances and cross-covariances are jointly equal to zero). For the PSID and SIME/DIME samples the MA(1) specification is rejected. For the NLS sample, however, the MA(1) specification is adequate. Given the sampling intervals of the PSID and NLS surveys, the processes generating the data in these two surveys may be of the same order. The process generating the SIME/DIME data is evidently of lower order.

The relatively simple covariance structures displayed in Tables IV-VII suggest that earnings and hours changes may be generated by some relatively parsimonious statistical model. We will examine three such models, in increasing order of complexity. The first of these is a simple stationary measurement error model. The second model adds a common nonstationary component to both earnings and hours changes. The third model adds an additional nonstationary component to the variances and contemporaneous covariances of earnings and hours changes. We will refer to these models as a pure measurement error model, a two-component model, and a three-component model, respectively.

There is a substantial body of recent research on the nature and consequences of measurement error in labor market survey data. ${ }^{20} \mathrm{~A}$ formulation that is consistent with this work is one in which the measurement error in reported earnings and hours consists of permanent individual effects together with purely transitory unsystematic measurement errors $\left(u_{i t}\right.$ and $\left.v_{i t}\right)$, with

$$
E\left[\begin{array}{l}
u_{i t} \\
v_{i t}
\end{array}\right]=0, \quad \operatorname{var}\left[\begin{array}{c}
u_{i t} \\
v_{i t}
\end{array}\right]=\left[\begin{array}{ll}
\sigma_{u u} & \sigma_{u v} \\
\sigma_{u v} & \sigma_{v v}
\end{array}\right]
$$

and

$$
\operatorname{cov}\left(u_{i t}, u_{i s}\right)=\operatorname{cov}\left(v_{i t}, v_{i s}\right)=\operatorname{cov}\left(u_{i t}, v_{i s}\right)=0, \quad t \neq s .
$$

This model permits arbitrary systematic bias in individual earnings and hours measures, but assumes serial independence in the transitory measurement errors. ${ }^{21}$

\footnotetext{
${ }^{19}$ By a stationary MA(2) representation, we mean that $\operatorname{cov}\left[\Delta \log g_{i t}, \Delta \log g_{i t-1}\right], \operatorname{cov}\left[\Delta \log h_{i t}\right.$, $\left.\Delta \log h_{i t-j}\right]$, and $\operatorname{cov}\left[\Delta \log g_{i t}, \Delta \log h_{i t-j}\right]$ are constant for all $t$ and are zero for $|j|>2$.

${ }^{20}$ See Mellow and Sider (1983), Freeman (1984), Abowd and Zellner (1985), Duncan and Hill (1985), Fuller and Chua (1985), and Poterba and Summers (1986).

${ }^{21}$ Note that any correlation of consecutive measurement errors in the levels of earnings or hours in this model arises from permanent individual effects, and is therefore restricted to be positive.
} 
On the assumption that deviations of reported earnings and hours from time means and experience effects consist solely of measurement error, equation (1) implies the following covariance structure for observed changes in experienceadjusted earnings and hours:

$$
\begin{aligned}
& \operatorname{cov}\left[\Delta \log \tilde{g}_{i t}, \Delta \log \tilde{g}_{i s}\right]= \begin{cases}2 \sigma_{u u}, & t=s, \\
-\sigma_{u u}, & |t-s|=1, \\
0, & |t-s|>1 ;\end{cases} \\
& \operatorname{cov}\left[\Delta \log \tilde{h}_{i t}, \Delta \log \tilde{h}_{i s}\right]= \begin{cases}2 \sigma_{v v}, & t=s, \\
-\sigma_{v v}, & |t-s|=1, \\
0, & |t-s|>1 ;\end{cases} \\
& \operatorname{cov}\left[\Delta \log \tilde{g}_{i t}, \Delta \log \tilde{h}_{i s}\right]= \begin{cases}2 \sigma_{u v}, & t=s, \\
-\sigma_{u v}, & |t-s|=1, \\
0, & |t-s|>1 .\end{cases}
\end{aligned}
$$

These equations imply that the first order autocorrelations of both earnings and hours are $-1 / 2$. They also imply that the ratio of the first-order cross-covariances of earnings and hours to their contemporaneous covariance is $-1 / 2$.

Equations (2a)-(2c) describe the entire covariance structure of earnings and hours changes in terms of only three parameters: $\sigma_{u u}, \sigma_{v v}$, and $\sigma_{u v}$. Row 4 of Table VIII displays the estimated goodness-of-fit statistics for this pure measurement error model fit to the variances, covariances, and first and second autocovariances of earnings and hours. Evidently, the four samples all display substantial departures from this model. Examination of the data in Tables IV-VII suggests two reasons that the pure measurement error model fails. The first is that first-order autocorrelations of earnings and hours are all less than $1 / 2$ in absolute value. The second reason is that the covariance structure of earnings and hours changes is far from time stationary.

Our second statistical model adds a single common component of variance of earnings and hours that can potentially account for both of these departures. In particular, consider a factor $z_{i t}$ that enters hours with a (normalized) coefficient of unity, and earnings with relative coefficient $\mu$, implying

$$
\begin{aligned}
& \Delta \log \tilde{g}_{i t}=\mu \Delta z_{i t}+\Delta u_{i t}, \\
& \Delta \log \tilde{h}_{i t}=\Delta z_{i t}+\Delta v_{i t} .
\end{aligned}
$$

Suppose that $\Delta z_{i t}$ follows an unrestricted second order moving average process, with

$$
E\left[\Delta z_{i t}\right]=0, \quad E\left[\Delta z_{i t}, \Delta u_{i t}\right]=E\left[\Delta z_{i t}, \Delta v_{i t}\right]=0,
$$

and

$$
\operatorname{cov}\left[\Delta z_{i t}, \Delta z_{i s}\right]=0 \text { for }|t-s|>2 \text {. }
$$


In this case, equations (3a) and (3b) imply:

$$
\operatorname{cov}\left[\Delta \log \tilde{g}_{i t}, \Delta \log \tilde{g}_{i s}\right]= \begin{cases}\mu^{2} \operatorname{var}\left[\Delta z_{i t}\right]+2 \sigma_{u u}, & t=s, \\ \mu^{2} \operatorname{cov}\left[\Delta z_{i t}, \Delta z_{i s}\right]-\sigma_{u u}, & |t-s|=1, \\ \mu^{2} \operatorname{cov}\left[\Delta z_{i t}, \Delta z_{i s}\right], & |t-s|=2, \\ 0, & \text { otherwise; }\end{cases}
$$

$$
\operatorname{cov}\left[\Delta \log \tilde{h}_{i t}, \Delta \log \tilde{h}_{i s}\right]= \begin{cases}\operatorname{var}\left[\Delta z_{i t}\right]+2 \sigma_{v v}, & t=s, \\ \operatorname{cov}\left[\Delta z_{i t}, \Delta z_{i s}\right]-\sigma_{v v}, & |t-s|=1, \\ \operatorname{cov}\left[\Delta z_{i t}, \Delta z_{i s}\right], & |t-s|=2, \\ 0, & \text { otherwise }\end{cases}
$$

$$
\operatorname{cov}\left[\Delta \log \tilde{g}_{i t}, \Delta \log \tilde{h}_{i s}\right]= \begin{cases}\mu \operatorname{var}\left[\Delta z_{i t}\right]-2 \sigma_{u v}, & t=s, \\ \mu \operatorname{cov}\left[\Delta z_{i t}, \Delta z_{i s}\right]-\sigma_{u v}, & |t-s|=1, \\ \mu \operatorname{cov}\left[\Delta z_{i t}, \Delta z_{i s}\right], & |t-s|=2, \\ 0, & \text { otherwise. }\end{cases}
$$

We refer to this model as a "two-component" model with a stationary measurement error component and a nonstationary common factor. Equations (4a)-(4c) imply a number of restrictions on the covariance matrix of earnings and hours changes. First, provided that $\operatorname{cov}\left[\Delta z_{i t}, \Delta z_{i t-1}\right]>0$, the first order autocorrelations of changes in earnings and hours are all between $-1 / 2$ and 0 . Second, if $\mu>0$ and $\operatorname{cov}\left[\Delta z_{i t}, \Delta z_{i t-1}\right]>0$, the ratio

$$
\frac{\operatorname{cov}\left[\Delta \log \tilde{h}_{i t}, \Delta \log \tilde{g}_{i t-j}\right]}{\operatorname{cov}\left[\Delta \log \tilde{h}_{i t}, \Delta \log \tilde{g}_{i t}\right]}
$$

is between $-1 / 2$ and 0 for $j=1,-1$. Third, the cross-covariance function of earnings and hours is symmetric:

$$
\operatorname{cov}\left[\Delta \log \tilde{g}_{i t}, \Delta \log \tilde{h}_{i t-s}\right]=\operatorname{cov}\left[\Delta \log \tilde{g}_{i t}, \Delta \log \tilde{h}_{i t+s}\right] .
$$

Tests of the latter restrictions are presented in row 5 of Table VIII. The symmetry restrictions are easily accepted in the NLS sample and the PSID sample without the SEO, and are marginally acceptable in the other two samples.

The sixth row of Table VIII displays the goodness-of-fit for the two-component model of equations (3a) and (3b), assuming that $\Delta z_{i t}$ follows a nonstationary second order moving average process. The estimated relative coefficient $\mu$ is presented in the seventh row of the table. All four samples provide substantial evidence against the two-component specification. There is, on the other hand, remarkable consistency across the data sets in the estimates of $\mu$. Maintaining a simple two-component structure, the nonmeasurement error component of variance in earnings and hours affects both variables more-or-less proportionally.

Close examination of the fit of the two-component model implied by equations (3a) and ( $3 b)$ suggests that the model has trouble fitting the pattern of nonsta- 


\section{TABLE VIII}

Summary of Goodness-of-Fit TeSTS For the EXPERIENCE-AdJuSTed Changes IN EARNINGS AND Hours for THE Panel STUdy OF INCOME DyNamics, THE National LONGITUdinal SURVEY OF MeN 45-59, AND THE SEATtLe/DenVer InCome MainTenanCe Experiment

\begin{tabular}{|c|c|c|c|c|}
\hline $\begin{array}{l}\text { Goodness-of-Fit } \\
\text { Statistic }\end{array}$ & $\begin{array}{l}\text { Annual } \\
\text { Data } \\
\text { from PSID }\end{array}$ & $\begin{array}{l}\text { Annual Data } \\
\text { from PSID } \\
\text { SEO Excluded }\end{array}$ & $\begin{array}{l}\text { Annual } \\
\text { Data } \\
\text { from NLS }\end{array}$ & $\begin{array}{c}\text { Semi-Annual } \\
\text { Data } \\
\text { from SIME/DIME }\end{array}$ \\
\hline \multicolumn{5}{|l|}{ 1. Nonstationary MA(2) ${ }^{\mathrm{b}}$} \\
\hline $\begin{array}{l}\text { a. Based on fourth moments } \\
\text { (probability value) } \\
\text { [degrees of freedom] }\end{array}$ & $\begin{array}{c}137.19 \\
(.062) \\
{[112]}\end{array}$ & $\begin{array}{c}148.91 \\
(.011) \\
{[112]}\end{array}$ & $\begin{array}{l}13.03 \\
(.367) \\
{[12]}\end{array}$ & $\begin{array}{l}74.98 \\
(.092) \\
{[60]}\end{array}$ \\
\hline b. Based on joint normality & 210.87 & 182.78 & 25.14 & 95.97 \\
\hline $\begin{array}{l}\text { (probability value) } \\
\text { [degrees of freedom in 1a] }\end{array}$ & $(.000)$ & $(.000)$ & $(.014)$ & $(.002)$ \\
\hline \multirow{2}{*}{ 2. Stationary MA(2) ${ }^{\mathrm{c}}$} & 143.69 & 133.85 & 68.84 & 100.70 \\
\hline & $(.000)$ & $(.001)$ & $(.000)$ & $(.000)$ \\
\hline [degrees of freedom] & [87] & [87] & [21] & [65] \\
\hline \multirow{2}{*}{ 3. Nonstati } & 50.39 & 55.46 & 15.11 & 39.95 \\
\hline & $(.020)$ & $(.006)$ & $(.235)$ & $(.022)$ \\
\hline [degrees of freedom] & [32] & [32] & [12] & [24] \\
\hline \multirow{3}{*}{$\begin{array}{l}\text { 4. Measurement error only }{ }^{c} \\
\text { (probability value) } \\
\text { [degrees of freedom] }\end{array}$} & 406.94 & 363.89 & 207.95 & 286.03 \\
\hline & $(.000)$ & $(.000)$ & $(.000)$ & $(.000)$ \\
\hline & [95] & [95] & [40] & [73] \\
\hline 5. Symmetric $\mathrm{MA}(2)^{\mathrm{c}}$ & 31.02 & 18.92 & 14.80 & 5.90 \\
\hline (probability value) & $(.020)$ & $(.333)$ & $(.039)$ & $(.434)$ \\
\hline [degrees of freedom] & [17] & [17] & [7] & [13] \\
\hline \multirow[t]{3}{*}{ 6. Two-c } & 261.15 & 239.51 & 124.13 & 229.36 \\
\hline & $(.000)$ & $(.000)$ & $(.000)$ & $(.000)$ \\
\hline & {$[68]$} & [68] & [28] & {$[52]$} \\
\hline \multicolumn{5}{|l|}{ 7. Estimate of coefficient $(\mu)$} \\
\hline for two-component model & 1.14 & 1.13 & 1.17 & .98 \\
\hline (standard error) & $(.09)$ & $(.09)$ & $(.12)$ & $(.06)$ \\
\hline \multirow{3}{*}{$\begin{array}{l}\text { 8. Three-component model } \\
\text { (probability value) } \\
\text { [degrees of freedom] }\end{array}$} & 62.26 & 72.00 & 31.38 & 59.98 \\
\hline & $(.026)$ & $(.014)$ & $(.026)$ & $(.014)$ \\
\hline & [48] & [48] & {$[16]$} & [36] \\
\hline \multicolumn{5}{|l|}{ 9. Estimate of coefficient $(\mu)$} \\
\hline for three-component model & 1.09 & 1.35 & 1.56 & 1.01 \\
\hline (standard error) & (.11) & $(.16)$ & $(.59)$ & $(.07)$ \\
\hline
\end{tabular}

\footnotetext{
${ }^{a}$ Data are sampled biennially.

${ }^{b}$ Versus the alternative hypothesis of a completely unrestricted covariance matrix. There are 210 total degrees of freedom (unique second-order moments) in the PSID, 55 in the NLS, and 136 in the SIME/DIME. 'Versus the alternative hypothesis of an unrestricted bivariate MA(2). There are 98 total degrees of freedom in the PSID, 43 in the NLS, and 76 in the SIME/DIME.
}

tionarity in the variances of earnings and hours over time. Equations (4a) and (4b) imply that the change in the variance of earnings between any two years, $\operatorname{var}\left[\Delta \log \tilde{g}_{i t}\right]-\operatorname{var}\left[\Delta \log \tilde{g}_{i s}\right]$, is strictly proportional to the change in the variance of hours between those years $\operatorname{var}\left[\Delta \log \tilde{h}_{i t}\right]-\operatorname{var}\left[\Delta \log \tilde{h}_{i s}\right]$. To relax this restriction, consider the earnings and hours equations

$$
\Delta \log \tilde{g}_{i t}=\mu \Delta z_{i t}+\Delta u_{i t}+\varepsilon_{1 i t}
$$

$$
\Delta \log \tilde{h}_{i t}=\Delta z_{i t}+\Delta v_{i t}+\varepsilon_{2 i t},
$$

where $E\left[\varepsilon_{1 i t}\right]=E\left[\varepsilon_{2 i t}\right]=0, \operatorname{var}\left[\varepsilon_{1 i t}\right]$, $\operatorname{var}\left[\varepsilon_{2 i t}\right]$, and $\operatorname{cov}\left[\varepsilon_{1 i t}, \varepsilon_{2 i t}\right]$ are unrestricted, 
and $\operatorname{cov}\left[\varepsilon_{j i t}, \varepsilon_{k i s}\right]=0$ for all $t \neq s$. This model implies the following structure for the variances and contemporaneous covariances of earnings and hours changes:

$$
\begin{aligned}
& \operatorname{var}\left[\Delta \log \tilde{g}_{i t}\right]=\mu^{2} \operatorname{var}\left[\Delta z_{i t}\right]+2 \sigma_{u u}+\operatorname{var}\left[\varepsilon_{1 i t}\right], \\
& \operatorname{var}\left[\Delta \log \tilde{h}_{i t}\right]=\operatorname{var}\left[\Delta z_{i t}\right]+2 \sigma_{v v}+\operatorname{var}\left[\varepsilon_{2 i t}\right], \\
& \operatorname{cov}\left[\Delta \log \tilde{g}_{i t}, \Delta \log \tilde{h}_{i t}\right]=\mu \operatorname{var}\left[\Delta z_{i t}\right]+2 \sigma_{u v}+\operatorname{cov}\left[\varepsilon_{1 i t}, \varepsilon_{2 i t}\right] .
\end{aligned}
$$

The expressions in equations (4a)-(4c) for the first and second order autocovariances and cross-covariances of earnings and hours remain valid. We refer to this as a "three-component model," consisting of a pure measurement error component, a common nonstationary component, and a transitory component affecting only the variances and contemporaneous covariances of earnings and hours. The effect of the third component is to unrestrict the variances and contemporaneous covariances of earnings and hours, while preserving a simple two-component structure for the autocovariances and higher-order cross covariances.

The eighth row of Table VIII displays the goodness-of-fit statistics for this three-component model. Corresponding estimates of the relative coefficient $\mu$ are presented in the ninth row of the table. The test statistics give only mild evidence of departures from the three-component structure. We conclude that this model gives a reasonable representation of the covariance structure of experienceadjusted earnings and hours changes for all four of our samples.

Surprisingly, the estimated relative coefficient of the common component of earnings and hours variation is similar in the two-component and three-component models. For the overall PSID sample and the SIME/DIME sample the estimates of $\mu$ from the three-component model are about equal to the estimates from the two-component model (and the standard errors are similar). For the NLS sample and the PSID sample excluding the SEO, the relative coefficient in the three-component model is larger than the estimate in the two-component model, but is not significantly different from 1 . Both the two-component and three-component models therefore suggest that the covariation of earnings and hours arises from changes in hours at fixed hourly wage rates, leading to proportional changes in earnings.

A final statistical issue we consider is the choice of estimator for the variance components models. Chamberlain $(1982,1984)$ has shown that the optimal minimum distance (OMD) estimator for these models weights the deviations between actual and predicted second movements by the inverse covariance matrix of the second moments. The estimates in Table VIII, on the other hand, are based on an equally weighted minimum distance (EWMD) estimator. (Appendix A lists the formulas used for both types of minimum distance estimators.) Table IX presents the optimal minimum distance estimates for the two-component and three-component models. Row 1a shows the goodness-of-fit statistics for the two-component model, which should be compared to the goodness-of-fit statistics in row 6 of Table VIII. Both the OMD and the EWMD test statistics reveal the lack of fit of the two-component model. However, as shown in row $1 \mathrm{~b}$ of Table IX, the OMD estimate of the relative coefficient $\mu$ can be seriously 
TABLE IX

Optimal Minimum Distance Estimates of Variance COMPONENTS MODEl AND SPECIFICATION TeSTS

\begin{tabular}{|c|c|c|c|c|}
\hline $\begin{array}{l}\text { Name of } \\
\text { Statistic }\end{array}$ & $\begin{array}{c}\text { Annual } \\
\text { Data } \\
\text { from PSID }\end{array}$ & $\begin{array}{l}\text { Annual Data } \\
\text { from PSID } \\
\text { SEO Excluded }\end{array}$ & $\begin{array}{c}\text { Annual } \\
\text { Data } \\
\text { from NLS }\end{array}$ & $\begin{array}{c}\text { Semi-Annual } \\
\text { Data } \\
\text { from SIME/DIME }\end{array}$ \\
\hline \multicolumn{5}{|l|}{ 1. Two-component model ${ }^{\mathrm{b}}$} \\
\hline a. Goodness-of-fit statistic & 261.12 & 173.07 & 94.49 & 227.19 \\
\hline (probability value) & $(.000)$ & $(.000)$ & $(.000)$ & $(.000)$ \\
\hline [degrees of freedom] & [68] & {$[68]$} & {$[28]$} & [52] \\
\hline b. Relative coefficient $(\mu)$ & 1.11 & 11.23 & 7.71 & 1.06 \\
\hline (standard error) & $(.03)$ & $(4.50)$ & (2.79) & $(.03)$ \\
\hline c. Specification test & .29 & $-^{c}$ & $-{ }^{c}$ & 1.54 \\
\hline (probability value) & $(.772)$ & & & $(.124)$ \\
\hline \multicolumn{5}{|l|}{ 2. Three-component model ${ }^{\mathrm{b}}$} \\
\hline a. Goodness-of-fit statistic & 61.38 & 69.23 & 31.21 & 58.86 \\
\hline (probability value) & $(.027)$ & $(.024)$ & $(.027)$ & $(.009)$ \\
\hline [degrees of freedom] & {$[48]$} & {$[48]$} & {$[16]$} & [36] \\
\hline b. Relative coefficient $(\mu)$ & .95 & .92 & .85 & 1.01 \\
\hline (standard error) & $(.08)$ & $(.12)$ & $(.29)$ & $(.03)$ \\
\hline c. Specification test & 1.68 & 3.86 & 1.38 & .002 \\
\hline (probability value) & $(.093)$ & $(.000)$ & $(.167)$ & $(.998)$ \\
\hline
\end{tabular}

\footnotetext{
${ }^{a}$ Data are sampled biennially.

${ }^{b}$ Versus the alternative hypothesis of an unrestricted bivariate MA(2). There are 98 total degrees of freedom in the PSID, 43 in the NLS, and 76 in the SIME/DIME.

${ }^{\mathrm{c}} \mathrm{Statistic}$ cannot be computed.
}

misleading in the case of an ill-fitting model. In two of the samples, the OMD estimate of $\mu$ from the two-component model is considerably in excess of unity. A simple specification test based on the difference between the OMD and EWMD parameter estimates of the two-component model is displayed in row 1 c. $^{22}$ The test statistic cannot be computed for two of the samples because the estimated sampling variance of the OMD estimator is actually larger than the estimated sampling variance of the EWMD estimator. ${ }^{23}$ In the other two surveys the test shows that the EWMD and OMD estimates are not very different.

For the three-component model the goodness-of-fit measures for the OMD estimates are comparable to the goodness-of-fit measures for the EWMD estimates. The estimates of the relative coefficient $\mu$ are also similar to the corresponding EWMD estimate and somewhat more precise. The specification tests (Table IX, row 2c) for the three-component model are satisfactory for three of the four samples, but provide some evidence against the three-component model for the PSID sample with the SEO excluded.

To summarize, the OMD and EWMD procedures give very similar measures of the goodness-of-fit of the two- and three-component models. For the three-com-

\footnotetext{
${ }^{22}$ This class of specification tests is suggested by Hausman (1978). Under the hypothesis that the model of the covariances is correct, the covariance matrix of the vector of differences between the EWMD and OMD parameter estimates is simply the difference in the estimated covariance matrices of EWMD and OMD parameter estimates.

${ }^{23}$ The variance of the OMD parameter estimate is necessarily smaller only when both variances are estimated at the same parameter values.
} 
ponent model, which provides an acceptable fit to the data, the procedures also give similar parameter estimates. On the other hand, for the two-component model, which fits relatively poorly, the EWMD and OMD parameter estimates differ for two of the four samples. We conclude that the efficiency gains from the optimally weighted minimum distance procedure, while noticeable, must be balanced against the greater risk of misleading estimates in the case of a poorly-fitting model.

\section{INTERPRETING THE COVARIANCE STRUCTURE OF EARNINGS AND HOURS CHANGES}

In this section we discuss a life-cycle labor supply interpretation of the covariance structure of earnings and hours changes. Since this covariance structure is adequately summarized by a simple three-component model, a statistically acceptable interpretation is one that includes only three components: measurement error, a common component of earnings and hours variation with a second-order moving average covariance structure, and a transitory component of cross-sectional variation in earnings and hours changes. All that is required is a mapping between the labor supply model and this simple statistical model. Since the focus of the labor supply model is on behavioral responses to individual productivity variation, we make the assumption that individual productivity variation is the source of systematic covariation between earnings and hours. According to the life-cycle model, changes in productivity affect earnings more than hours. As we noted in Section I, however, the estimated relative coefficient $(\mu)$ of the empirically determined common component of earnings and hours variation is approximately unity. This suggests that the covariation of earnings and hours changes does not arise from labor supply responses to productivity variation. Rather, most of the covariation of earnings and hours occurs at fixed hourly wage rates.

The life-cycle supply model is a standard tool in the analysis of individual earnings and hours data. ${ }^{24}$ The starting point for our presentation of this model is the labor supply equation

$$
\log h_{i t}=a_{i t}+\eta \log \theta_{i t}+\eta \log \lambda_{i t},
$$

where $h_{i t}$ represents the hours choice of individual $i$ in period $t, a_{i t}$ is an intercept shift associated with individual and time-period specific preference variation, $\theta_{i t}$ represents the (unobservable) hourly wage rate for individual $i$ in period $t, \lambda_{i t}$ represents the marginal utility of consumption for $i$ in period $t$, and $\eta$ is a fixed parameter that represents the intertemporal substitution elasticity. ${ }^{25}$ The interpretation of equation (7) is as follows: for given preferences and fixed marginal utility of consumption, the elasticity of hours with respect to wages is

\footnotetext{
${ }^{24}$ See for example Ghez and Becker (1975), Heckman (1976), MaCurdy (1981), and Altonji (1986).

${ }^{25}$ Equation (7) may be derived directly from the first-order conditions for maximizing an additively separable intertemporal utility function with the within-period objective $u(c)-v(h)$, where $c$ and $h$ represent consumption and hours choices in the period, and $v(h)$ has the form $A h^{b}$, where $b=(1+\eta) / \eta$. See MaCurdy $(1981$, p. 1064).
} 
the strictly positive number $\eta$. The relation between $\eta$ and other conceptual measures of the elasticity of hours with respect to wage rates is described by MaCurdy (1981).

In addition to the restriction on the sign of $\eta$, the life-cycle labor supply model restricts the time path of the marginal utility of consumption $\left(\lambda_{i t}\right)$. Specifically, the expected value of $\lambda_{i t+1}$ in period $t$ is related to $\lambda_{i t}$ by

$$
E_{t}\left(\lambda_{i t+1}\right)=\frac{1+\rho}{1+r_{t}} \lambda_{i t}
$$

where $\rho$ is the subjective discount rate in the intertemporal objective function (assumed to be constant over time and across individuals), and $r_{t}$ is the real rate of return on assets between period $t$ and period $t+1$ (assumed to be constant across assets and individuals and known in period $t$ ) ${ }^{26}$ With perfect foresight, equation (8) implies that $\log \lambda_{i t}$ acts as a fixed effect for individual $i$ in the labor supply equation (7). ${ }^{27}$ More generally, however, if $\phi_{i t}$ is defined as the one period ahead prediction error in the logarithm of the marginal utility of consumption $\left(\phi_{i t} \equiv \log \lambda_{i t}-E_{t-1}\left(\log \lambda_{i t}\right)\right)$ then equation (8) implies

$$
\log \lambda_{i t}=\log \left(\frac{1+\rho}{1+r_{t}}\right)+\log \lambda_{i t-1}+\phi_{i t}+\zeta_{i t},
$$

where $\zeta_{i t}=-\log E_{t-1}\left(\exp \left(\phi_{i t}\right)\right) .{ }^{28}$ If variation in $\zeta_{i t}$ is negligible, as we shall assume, then equation (9) has the straightforward interpretation that the individual-specific component of the change in the marginal utility of consumption is a (serially uncorrelated) prediction error.

Assuming that labor earnings represent the product of average hourly earnings and hours worked, the labor supply equation (7) implies

$$
\log g_{i t}=a_{i t}+(1+\eta) \log \theta_{i t}+\eta \log \lambda_{i t},
$$

where $g_{i t}$ denotes the earnings of individual $i$ in period $t$. Equations (7), (9), and (10) together describe the implications of the life-cycle labor supply model for changes in individual earnings and hours over time.

As we noted in Section 1, however, there is widespread evidence that survey measures of earnings and hours contain measurement error. We assume that this measurement error takes the form of equations (1a) and (1b). Combining equations (1a), (1b), (7), (9), and (10), the life-cycle labor supply model implies that observed changes in earnings and hours follow

$$
\begin{aligned}
& \Delta \log g_{i t}=\eta \log \left(\frac{1+\rho}{1+r_{t}}\right)+\Delta a_{i t}+(1+\eta) \Delta \log \theta_{i t}+\eta \phi_{i t}+\Delta u_{i t}, \\
& \Delta \log h_{i t}=\eta \log \left(\frac{1+\rho}{1+r_{t}}\right)+\Delta a_{i t}+\eta \Delta \log \theta_{i t}+\eta \phi_{i t}+\Delta v_{i t},
\end{aligned}
$$

\footnotetext{
${ }^{26}$ See, for example, Browning, Deaton, and Irish (1985). Versions of equation (8) also appear in tests of the permanent income hypothesis (Hall (1978)).

${ }^{27}$ See Ashenfelter and Ham (1979), MaCurdy (1981), and Browning, Deaton, and Irish (1985).

${ }^{28}$ See MaCurdy $(1983$, p. 274) for a similar derivation.
} 
where $u_{i t}$ and $v_{i t}$ are the transitory measurement errors in earnings and hours, respectively. Deviations of individual earnings and hours changes from a multivariate regression on time period indicator variables and individual experience therefore follow

$$
\begin{aligned}
& \Delta \log \tilde{g}_{i t}=\Delta \tilde{a}_{i t}+(1+\eta) \Delta \log \tilde{\theta}_{i t}+\eta \phi_{i t}+\Delta u_{i t}, \\
& \Delta \log \tilde{h}_{i t}=\Delta \tilde{a}_{i t}+\eta \Delta \log \tilde{\theta}_{i t}+\eta \phi_{i t}+\Delta v_{i t},
\end{aligned}
$$

where $\Delta \tilde{a}_{i t}$ and $\Delta \log \tilde{\theta}_{i t}$ represent experience-adjusted taste and productivity variation, respectively, and $\phi_{i t}$ is now interpreted as the deviation of the unanticipated change in $\lambda_{i t}$ from the mean change across individuals in period $t .^{29}$

There are a number of alternative mappings between the variance components in equation (12a) and (12b) and the statistical models of the previous Section. The life-cycle labor supply model, however, emphasizes the role of individual productivity in earnings and hours determination. Accordingly, we identify the common component of earnings and hours variation with individual productivity variation. Specifically, we assume that individual productivity variation around a deterministic experience profile, $\Delta \log \tilde{\theta}_{i t}$, follows a nonstationary second-order moving average process. According to the life-cycle labor supply model, this component of variance enters earnings and hours with coefficients of $\eta$ and $(1+\eta)$, respectively. Normalizing the coefficient on hours to unity, the relative coefficient of productivity variation on earnings is $(1+\eta) / \eta>1$.

The statistical identification of productivity variation as the common component of earnings and hours variation (i.e., as the second component in the empirical model in Section 1) requires assumptions on the covariance structure of the other two components in earnings and hours-preference variation $\Delta \tilde{a}_{i t}$, and changes in the marginal utility of consumption $\phi_{i t}$. The covariance structure of $\phi_{i t}$ is restricted by the hypothesis that changes in the marginal utility of consumption are unanticipated; hence $\operatorname{plim} \sum_{t} \phi_{i t} \phi_{i t-j}=0$ for $j \neq 0$. In cross-section, however, $\operatorname{cov}\left[\phi_{i t}, \phi_{i t-j}\right]$ may be nonzero if individuals systematically vary in the response of $\lambda_{i t}$ to unanticipated aggregate shocks.

To develop this point, observe that $\phi_{i t}$ depends on new information about the idiosyncratic productivity component $\tilde{\theta}_{i t}$, as well as new information about aggregate variables (aggregate productivity, interest rates). Suppose that aggregate information is summarized by a scalar $y_{t}$, and consider the linear projection (over time for a fixed individual)

$$
\phi_{i t}=c_{i}\left(\tilde{\theta}_{i t}-E_{t-1} \tilde{\theta}_{i t}\right)+d_{i}\left(y_{t}-E_{t-1} y_{t}\right)+e_{i t} .
$$

The possibility that unexpected aggregate information can affect workers differently is captured by the individual-specific coefficient $d_{i}$. Using this equation, $\operatorname{cov}\left(\phi_{i t}, \phi_{i s}\right)=\operatorname{var}\left[d_{i}\right]\left(y_{t}-E_{t-1} y_{t}\right)\left(y_{s}-E_{s-1} y_{s}\right)+\operatorname{cov}\left[e_{i t}, e_{i s}\right]$. If dispersion in $d_{i}$ is large, we would expect to observe nonvanishing covariances between changes in earnings and hours from years with larger aggregate shocks, regardless of the

\footnotetext{
${ }^{29}$ Economy wide preference and productivity effects are also removed from $\Delta \tilde{a}_{i t}$ and $\Delta \log \tilde{\theta}_{i t}$.
} 
gap between these years. Since there is no evidence of this phenomenon in Tables IV-VII, we proceed under the assumption that unanticipated aggregate shocks generate homogeneous shifts in the marginal utility of consumption. In this case, the projection error $e_{i t}$ is only a function of $\left(\tilde{\theta}_{i t}-E_{t-1} \tilde{\theta}_{i t}\right)$, and the life-cycle model implies $\operatorname{cov}\left[\phi_{i t}, \phi_{i t-j}\right]=0$ for $j \neq 0$. Thus, changes in the marginal utility of consumption only contribute to the variances and the contemporaneous covariances of earnings and hours changes. Changes in the marginal utility of consumption are therefore absorbed by the third component in the empirical model of Section 1.

The covariance structure of preference variation, however, is unrestricted by the theory of life-cycle labor supply. Obviously, if preference variation has the same covariance structure as productivity variation, then the relative variance contribution of these two components are not separately identifiable. The most general model of preference variation that can be mapped into the first and third components of the three-component model developed in Section 1 is one consisting of "transitory" preference shocks $\nu_{i t}$ and "permanent" preference shocks $\zeta_{i t}$ with the properties

$$
\begin{array}{ll}
\operatorname{var}\left[\nu_{i t}\right]=\alpha, \quad \operatorname{cov}\left[\nu_{i t}, \nu_{i s}\right]=0, & s \neq t, \\
\operatorname{cov}\left[\zeta_{i t}-\zeta_{i t-1}, \zeta_{i t-j}-\zeta_{i t-j-1}\right]=0, & j>0 .
\end{array}
$$

Equation (13a) states that transitory preference shocks are serially uncorrelated with a constant variance over time. Equation (13b) states that changes in the permanent preference shock are serially uncorrelated.

Under these assumptions, the contribution of preference variation to the covariance structure of experience-adjusted changes in earnings and hours is

$$
\Delta \tilde{a}_{i t}=\Delta \nu_{i t}+\Delta \zeta_{i t} .
$$

By construction, the component of variance in earnings and hours changes due to transitory preference variation is indistinguishable from the component due to transitory measurement errors. ${ }^{30}$ We therefore subsume $\Delta \nu_{i t}$ in the bivariate measurement error process. The component of variance due to permanent preference shocks, on the other hand, contributes only to the variances and contemporaneous covariances of changes in earnings and hours. Taste variation of this form is absorbed in the third component of the model in the previous section.

To summarize, if preference variation takes the form of either purely transitory or fully permanent changes in tastes for leisure, then its contribution to the covariance structure of earnings and hours is absorbed by the first and third components of the statistical model in the previous section. Under this assumption, we can uniquely identify individual productivity variation with the second

\footnotetext{
${ }^{30}$ The bivariate process $\left(\Delta v_{i t}+\Delta u_{i t}, \Delta v_{i t}+\Delta v_{i t}\right)$ has the same covariance structure as the pure measurement error process $\left(\Delta u_{i t}, \Delta v_{i t}\right)$, since the correlation between the measurement errors in earnings and hours is unrestricted.
} 
TABLE X

ESTIMATES OF THE ELASTICITY OF INTERTEMPORAL LABOR SUPPLY BaSED ON THE ESTIMATED RELATIVE COEFFICIENTS FROM THE VARIANCE COMPONENTS MODELS FOR EARNINGS AND HOURS CHANGES ${ }^{\mathrm{a}}$

\begin{tabular}{|c|c|c|c|c|}
\hline $\begin{array}{l}\text { Structure and } \\
\text { Estimation Method }\end{array}$ & $\begin{array}{l}\text { Annual } \\
\text { Data } \\
\text { from PSID }\end{array}$ & $\begin{array}{l}\text { Annual Data } \\
\text { from PSID } \\
\text { SEO Excluded }\end{array}$ & $\begin{array}{l}\text { Annual } \\
\text { Data } \\
\text { from NLS }\end{array}$ & $\begin{array}{c}\text { Semi-Annual } \\
\text { Data } \\
\text { from SIME/DIME }\end{array}$ \\
\hline
\end{tabular}

1. Two-component model

a. Equally weighted minimum distance (standard error)

b. Optimal minimum distance (standard error)

2. Three-component model

a. Equally weighted minimum distance (standard error)

$\begin{array}{cccc}7.27 & 7.62 & 5.88 & -55.55 \\ (4.81) & (4.97) & (4.11) & (78.98) \\ 8.83 & .10 & .15 & 16.67 \\ (2.63) & (.04) & (.06) & (7.23)\end{array}$

b. Optimal minimum distance -19.11 (standard error)

(28.45)

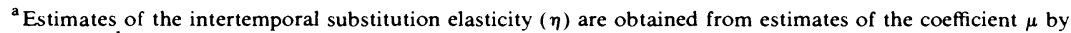
$\eta=(\mu-1)^{-1}$. Standard errors are obtained by the delta method.

${ }^{\mathrm{b}}$ Data are sampled biennially.
}

component. The relative coefficient $\mu$ in equation (3a) can therefore be interpreted as an estimate of $(1+\eta) / \eta$, where $\eta$ is the intertemporal labor supply elasticity.

The implied estimates of $\eta$ corresponding to the estimates of $\mu$ in Tables VIII and IX are summarized in Table X. The point estimates of $\eta$ are extremely varied and imprecise, ranging from -55.6 (associated with an estimate of $\mu=.98$ for the two-component model applied to the SIME/DIME sample) to 125.0 (associated with an estimate of $\mu=1.01$ for the three-component model applied to the same data). The problem with these estimates of $\eta$ is that the estimates of $\mu$ are so close to unity. If $\mu=1$, changes in hours occur at fixed wage rates, suggesting in a labor supply framework that leisure is extremely substitutable over time.

There are a number of alternative interpretations of the data, however. One possibility is that the shared component of earnings and hours variation is mainly preference variation which enters with equal factor loadings in earnings and hours. In our view, this interpretation implies that the life-cycle labor supply model is vacuous: movements in earnings and hours restricted by the theory are empirically negligible. Another possibility is that earnings differ systematically from the level implied by the life-cycle labor supply model because of long term contracts between firms and workers. In previous work (Abowd and Card (1987)) we have derived the implications of a stylized contract in which observed earnings represent optimal consumption. The prediction of this model is that productivity variation affects earnings less than hours, for workers who are observed in the same contract over time. Looking at employees who are observed on the same job over extended periods, however, we have found that estimates of $\mu$ tend to be close to unity. The relative variation of earnings and hours changes 
among workers with the same employer over time is therefore inconsistent with the pattern of earnings smoothing implied by simple implicit contract models.

The relation between earnings and hours predicted by contracting models, however, depends critically on the assumption that workers can observe their own productivity. Grossman and Hart (1981) and many subsequent authors present contracting models in which earnings and hours satisfy an "incentivecompatibility" constraint - namely, that the firm truthfully declares the realized state of productivity. In a simple model where firm profits are proportional to $\theta_{i t} h_{i t}-g_{i t}$, and the contractual earnings and hours functions are differentiable functions of productivity $\left(h\left(\theta_{i t}\right)\right.$ and $g\left(\theta_{i t}\right)$, respectively), incentive-compatibility is characterized by the condition $\theta_{i t} h^{\prime}\left(\theta_{i t}\right)=g^{\prime}\left(\theta_{i t}\right) \geqslant 0 .{ }^{31}$ This condition implies that changes in productivity lead to greater proportional changes in earnings than hours. ${ }^{32}$ Thus incentive-compatible asymmetric information contracting models have the same predictions for the relative effect of productivity variation on earnings and hours changes as the life-cycle labor supply model.

A third interpretation of the covariance structure of earnings and hours changes is that individual wage rates are essentially fixed, and that proportional movements in earnings and hours occur because of changes in the demand for individual labor services. While this view is potentially consistent with the covariance of earnings and hours at the individual level, further research is clearly required to distinguish it from a model of pure taste variation. ${ }^{33}$

\section{CONCLUSION}

In this paper we have examined the covariance structure of earnings and hours changes for adult males in four samples drawn from three longitudinal surveys. There is remarkable similarity among the estimated covariance structures. In each of the four samples, changes in earnings and hours are positively correlated, although the implied correlations between changes in hours and changes in average hourly earnings are negative. Consecutive changes in earnings and hours, on the other hand, are strongly negatively correlated, suggesting the presence of random measurement error in the levels of both variables. Finally, changes in earnings and hours more than two periods apart are uncorrelated in all four samples.

\footnotetext{
${ }^{31}$ This condition may be derived by comparing profits when productivity is $\theta$ and the firm falsely declares $\theta^{*}$, and profits when productivity is $\theta$ and the firm truthfully declares $\theta$. Topel and Welch (1986) present an alternative derivation that considers earnings as a function of hours. In their framework, the incentive compatibility constraint is written as $(d g / d h)=\left(U_{l} / U_{c}\right)$, where $U_{l}$ and $U_{c}$ are the derivatives of the workers's utility function with respect to leisure and consumption in productivity state $\theta$. For the case where output is $\theta h(\theta)$, their first order condition for $h(\theta)$ gives $U_{l} / U_{c}=\theta$, implying $(d g / d h)=\theta$, or equivalently, $g^{\prime}(\theta)=\theta h^{\prime}(\theta)$.

${ }^{32}$ The condition $\theta h^{\prime}(\theta)=g^{\prime}(\theta)$ implies that
}

$$
\frac{d \log g(\theta)}{d \log \theta}=[1+\pi(\theta) / g(\theta)] \frac{d \log h(\theta)}{d \log \theta},
$$

where $\pi(\theta)=\theta h(\theta)-g(\theta) \geqslant 0$.

${ }^{33}$ One test of equilibrium fixed wage models is developed by Abowd and Ashenfelter (1981). 
We find that a components-of-variance model with three sources of earnings and hours variation successfully summarizes the covariance structure of the data from each survey. The first component consists of time-stationary serially uncorrelated measurement error. The second is a shared component of earnings and hours variation that affects the contemporaneous variances and first and second order covariances of both earnings and hours. The third is a time-varying component that affects only the variances and contemporaneous covariances of earnings and hours changes.

We then present an interpretation of this components-of-variance structure in terms of a life-cycle labor supply model. We associate the shared component of earnings and hours variation with individual productivity variation, which, according to the life-cycle model, affects earnings more than hours. Our empirical results for all four samples, however, suggest that earnings and hours covary proportionally. This casts doubt on the labor supply interpretation of earnings and hours variation, and suggests that most changes in earnings and hours occur at fixed hourly wage rates.

NYS School of Industrial and Labor Relations and Johnson Graduate School of Management, Cornell University, Ithaca, NY 14851, U.S.A.,

and

Department of Economics, Princeton University, Princeton, NJ 08544, U.S.A.

Manuscript received December, 1985; final revision received June, 1988.

\section{APPENDIX A \\ STATISTICAL METHODS}

In this appendix we summarize the estimation and inference procedures used throughout the paper. The basic unit of data for each individual in a particular data set is the vector of experienceadjusted changes in earnings and hours. If we denote these by $\Delta \log \tilde{g}_{i t}$ and $\Delta \log \tilde{h}_{i t}$, then the data vector is

$$
\tilde{y}_{i}=\left[\begin{array}{c}
\Delta \log \tilde{g}_{i 1} \\
\vdots \\
\Delta \log \tilde{g}_{i T} \\
\Delta \log \tilde{h}_{i 1} \\
\vdots \\
\Delta \log \tilde{h}_{i T}
\end{array}\right]
$$

which has dimension $2 T$, where $T$ is the number of changes observed in the data set. The elements of $\tilde{y}_{i}$ have zero mean by construction. Let $C=(1 / N) \sum_{i}\left(\tilde{y}_{i} \tilde{y}_{i}^{\prime}\right)$ represent the covariance matrix of these changes. Estimates of $C$ are presented for the four samples in Tables IV-VII.

The models we estimate are models for $C$. Let $m$ represent a vector whose elements are the distinct elements of $C$. Since $C$ is symmetric, there are only $2 T(2 T+1) / 2$ elements in $m$. In the PSID, this corresponds to 210 elements; in the NLS, 55 elements, and in the SIME/DIME, 136. Conformably with $m$, let $m_{i}$ represent the distinct elements of the individual cross-products matrix $\tilde{y}_{i} \tilde{y}_{i}^{\prime}$. Then $m=(1 / N) \sum_{i} m_{i}$. 
The variance matrix $V$ of the vector of covariance elements is estimated by computing the cross-sectional variance of $m_{i}$ in the usual manner:

$$
V=\frac{1}{N} \sum_{i}\left(m_{i}-m\right)\left(m_{i}-m\right)^{\prime} .
$$

A typical element of $V$ is $V_{u v}=\operatorname{cov}\left(m_{u}, m_{v}\right)$. If $m_{u}=\operatorname{cov}\left(\Delta \log \tilde{g}_{i t}, \Delta \log \tilde{h}_{i t-j}\right)$ and $m_{v}=$ $\operatorname{cov}\left(\Delta \log \tilde{g}_{i s}, \Delta \log \tilde{g}_{i s-k}\right)$, then

$$
\begin{aligned}
V_{u v}= & \frac{1}{N} \sum_{i}\left[\left(\Delta \log \tilde{g}_{i t}-\Delta \log \tilde{g}_{t}\right)\left(\Delta \log \tilde{h}_{i t-j}-\Delta \log \tilde{h}_{t-j}\right)-m_{u}\right] \\
& \times\left[\left(\Delta \log \tilde{g}_{i s}-\Delta \log \tilde{g}_{s}\right)\left(\Delta \log \tilde{g}_{i s-k}-\Delta \log \tilde{g}_{s-k}\right)-m_{v}\right],
\end{aligned}
$$

where $\Delta \log \tilde{g}_{t}$ represents the sample average change in the logarithm of earnings in period $t$. Let $Q$ represent the matrix of uncorrected fourth moments of $\tilde{y}_{i}$ :

$$
Q=\frac{1}{N} \sum_{i} m_{i} m_{i}^{\prime}
$$

$Q$ and $V$ are related by $V=Q-m m^{\prime}$.

Under fairly general conditions (see Chamberlain $(1982,1984)$ ) independence of the $\tilde{y}_{i}$ implies that the sample mean of $m_{i}$ has an asymptotic normal distribution:

$$
\sqrt{N}(m-\mu) \stackrel{a}{\sim} N\left(O, V^{*}\right),
$$

where $\mu$ is the expectation of $m_{i}$ (i.e., the true covariance matrix of earnings and hours changes) and $V^{*}=E\left(m_{i} m_{i}^{\prime}\right)-E\left(m_{i}\right) E\left(m_{i}^{\prime}\right)$.

Consider a model for the vector of covariance elements that depends on a lower-dimensional parameter vector $b$, say $m=f(b)$. Several estimators are available for $b$ : among these are the optimal minimum distance (OMD) estimator $b^{0}$, which minimizes $(m-f(b))^{\prime} V^{-1}(m-f(b))$, and the equally weighted minimum distance (EWMD) (or least squares) estimator $b^{e}$, which minimizes $(m-f(b))^{\prime}$ $\cdot(m-f(b))$. Optimality of the former estimator is discussed in Chamberlain (1984).

For the OMD estimator, inference is based on the fact that under the hypothesis of a correct specification, the minimized quadratic form

$$
N \cdot\left(m-f\left(b^{0}\right)\right)^{\prime} V^{-1}\left(m-f\left(b^{0}\right)\right)
$$

has an asymptotic $\chi^{2}$ distribution with degrees of freedom equal to the difference between the dimension of $m$ and the rank of the Jacobian matrix $F(b)=\partial f(b) / \partial b$, evaluated at $b^{*}$, the true value of $b$. (See Chamberlain (1984).)

If the model for $m$ has the special form

$$
m=\left[\begin{array}{l}
m_{1} \\
m_{2}
\end{array}\right]=F(b)=\left[\begin{array}{l}
b \\
0
\end{array}\right],
$$

in which only the last $k$ elements of $m$ are restricted to zero, it is straightforward to show that the minimized quadratic form reduces to

$$
N \cdot m_{2}^{\prime} V_{22}^{-1} m_{2},
$$

where $V_{22}$ is the block of $V$ corresponding to the elements in $m$ that are restricted to zero. This is the $\chi^{2}$ test for $m_{2}=0$.

For an estimator $b^{A}$ that minimizes an arbitrary quadratic form

$$
(m-f(b))^{\prime} A(m-f(b)),
$$

where $A$ is a positive definite matrix, Newey (1985) shows that the quadratic form

$$
N \cdot\left(m-f\left(b^{A}\right)\right)^{\prime} R^{-}\left(m-f\left(b^{A}\right)\right)
$$

has an asymptotic $\chi^{2}$ distribution. Here $R^{-}$is a generalized inverse of the matrix $R=P V P^{\prime}$, where $P=I-F\left(F^{\prime} A F\right)^{-1} F^{\prime} A$, and $F$ represents the Jacobian matrix of $f$ evaluated at $b^{A}$. Newey (1985) suggests a generalized inverse of $R$ of the form $S\left(S^{\prime} R S\right)^{-1} S^{\prime}$, where $S$ is a selection matrix of rank 
equal to the difference between the dimension of $m$ (the number of moments fit) and the rank of $F$. In general, for a nonlinear model, the value of the quadratic form depends on the exact generalized inverse selected for $R$. In the case of a linear model, however, the value of the quadratic form is invariant, and in the simple case of testing the restrictions $m_{2}=0$, with no additional restrictions on $m_{1}$, the value of the quadratic form can be shown to equal $N \cdot m_{2}^{\prime} V_{22}^{-1} m_{2}$. Tests of zero restrictions are therefore invariant to the choice of OMD or any arbitrary minimum distance estimator.

Our procedure is to first test for zero restrictions and then to work with the nonzero covariances of earnings and hours. In the notation of the previous discussion, once we have accepted the hypothesis $m_{2}=0$, we estimate models for $m_{1}$ and use the covariance matrix $V_{11}$ for inference. This procedure is not fully efficient, since better estimates of the unrestricted elements of $m$ can be formed by taking into account the fact that certain other elements of $m$ (i.e. those in $m_{2}$ ) are zero.

\section{APPENDIX B \\ DATA DESCRIPTION}

For the Panel Study of Income Dynamics we used an extract from the thirteen year merged individual tape distributed through the Inter-University Consortium for Political and Social Research and documented by the Survey Research Center of the Institute for Social Research (1981 and previous years). Our sample consisted of all males on the thirteen year merged individual tape with complete age and schooling data who were continuously heads of household from wave III to wave XIII of the survey and who reported nonzero annual labor earnings and annual hours in each of the 11 waves. Individuals with average hourly earnings greater than $\$ 100$ or reported annual hours greater than $\mathbf{4 6 8 0}$ were excluded from our samples.

The following is a description of the PSID variables used. Numbers like Vxxxx refer to the variable numbers of the Survey Research Center codebooks for the thirteen year merged individual tape.

ANNUAL EARNINGS: The variables used were: V1196, V1897, V2498, V3051, V3463, V3863, V5031, V6174, V6767, and V7413. These correspond to Survey Research Center's computed values for the head of household's total labor income in the calendar year before the survey.

ANNUAL HOURS: The variables used were V1138, V1839, V2439, V3027, V3423, V3823, V4332, V5232, V5731, V6336, and V6934. These correspond to Survey Research Center's computed value for the head of household's annual hours worked in the calendar year before the survey.

SEX: The variables used were V7492, V7509, V7526, V7547, V7561, V7576, V7601, V7653, and V7714. Only males were included.

HEAD OF HOUSEHOLD: The variables used were V7490, V7507, V7524, V7545, V7559, V7574, V7599, V7624, V7651, V7685, and V7712. Only heads of household for all eleven waves from wave III to wave XIII were included.

SCHOOLING: The variables used were V0313, V0794, V1485, V2197, V2838, V3241, V3663, V4198, V5074, V5647, V6194, V6787, and V7433. Maximum completed schooling was determined by examining all schooling variables. This was the schooling level used.

AGE: The variables used were V7460, V7476, and V7491. Age was determined for the first year an individual entered the sample, then adjusted to age in 1969.

SEO: The variable used was V132. The SEO subsample has position values for V132.

For the National Longitudinal Survey of Men 45-59, 1966 to 1975, we used an extract from the public use data file release 75A distributed through the Inter-University Consortium for Political and Social Research and documented by the Center for Human Resource Research $(1977,1980)$. We used data from survey years 1966, 1967, 1971, 1973, and 1975. Annual data from the survey year 1966 refer to calendar year 1965. Subsequent annual data refer to the twelve months preceding the actual interview - approximately June, 1966 to May, 1967 for the 1967 survey and approximately July of the previous year to June of the survey year for the subsequent surveys. Our sample consisted of all males who have valid age and schooling data and reported nonzero annual earnings and annual hours for the years we studied.

The following is a description of the NLS variables used. Numbers like Vxxxx refer to the Center for Human Resource Research codebook variable numbers (not the reference numbers) for the release $75 \mathrm{~A}$ public use tape. (Some variables are assigned two consecutive variable numbers.)

ANNUAL EARNINGS: The variables used were V0263-4, V0784-5, V1280-1, V3166-7, V2528-9, V2685-6.

ANNUAL HOURS: The variables used were hours per week: V0082, V0660, V1128, V1581, V2520, V2675, and weeks per year: V0589, V1022, V1168, (V2421 with V2461), V2519, V2674. For 
the 1971 survey we recoded the weeks worked variable, which refers to weeks worked since the last interview, into weeks worked in the last year by dividing the number of weeks worked since the last interview (V2421) by the number of weeks since the last interview (V2461) and multiplying by fifty-two.

AGE: The variable used was V0024, age in 1966.

EDUCATION: The variable used was V0611, highest grade completed.

For the Seattle-Denver Income Maintenance Experiment, we used an extract of male household heads for the Work Impact File, distributed by the National Archives Machine Readable Records Branch. Documentation for this file is provided on National Archive Microfiche M1363, "Seattle/ Denver Income Maintenance Experiment, 1970-78" (Fiche number 6, "Work Impact Record Layout"). We used male heads of dual-headed households from the nonexperimental group who had no change in treatment status during the experimental period, who were between 22 and 59 years old at enrollment into the experiment, and who reported nonzero labor earnings and between 20 and 2500 hours worked in each of the two pre-enrollment half-year periods and each of the first six post-enrollment half-year periods.

We used the following variables on the Work Impact File (referenced by their variable numbers in the codebook as Vxxx.)

EARNINGS: The variables used were V91, V92, and V779-V784.

HOURS: The variables used were V97, V98, and V803-V808.

HEAD STATUS: Heads of dual-headed households were determined by their "Group-level" status (variable V2). Heads of dual-headed households are recorded with "Group-level" 2, 4, or 6.

TREATMENT STATUS: Heads of control group families were determined by their "Financial Treatment Level" (variable V4). Heads of control-group families are recorded with "Financial Treatment Level" 0.

ENTRY DATE: Date of assignnent into the experiment is recorded as variable V7. Entry dates were coded into 8 quarterly intervals, starting with dates before January $1,1971$.

CHANGE IN TREATMENT STATUS: Change in treatment status between consecutive half-year periods is coded as V25, and V623-V635.

AGE: Age of male head in the month prior to enrollment, V27.

EDUCATION: Highest grade completed by male head at enrollment, V28.

\section{REFERENCES}

ABOWd, John M., AND Orley ASHENFelter (1981): “Anticipated Unemployment, Temporary Layoffs and Compensating Wage Differentials," Studies in Labor Markets, ed. by Sherwin Rosen. Chicago: University of Chicago Press (for the National Bureau of Economic Research), pp. 141-170.

ABowd, John M., and David CARD (1986): “On the Covariance Structure of Earnings and Hours Changes," National Bureau of Economic Research Working Paper Number 1832.

- (1987): "Intertemporal Labor Supply and Long Term Employment Contracts," American Economic Review, 77, 50-68.

Abowd, JoHn M., AND ARNold Zellner (1985): “Estimating Gross Labor Force Flows," Journal of Business and Economic Statistics, 3, 254-283.

AltoNJI, JOSEPH A. (1986): "Intertemporal Substitution in Labor Supply: Evidence from Micro Data," The Journal of Political Economy, 94, S176-S215.

ANDERSON, T. W. (1958): Introduction to Multivariate Statistical Analysis. New York: John Wiley.

AShenfelter, OrLey, AND JohN C. HAM (1979): "Education, Unemployment, and Earnings," Journal of Political Economy, 87, S99-S116.

Browning, Martin, Angus Deaton, and Margaret Irish (1985): “A Profitable Approach to Labor Supply and Commodity Demand Over the Life-cycle," Econometrica, 53, 503-543.

Center fOR HUman Resource ReSEARCh (1977): National Longitudinal Survey of Men 45-59, 1966 to 1976: Codebook. Columbus, Ohio: The Ohio State University.

(1981): The National Longitudinal Surveys Handbook. Columbus, Ohio: The Ohio State University.

Chamberlain, Gary (1978): "Omitted Variable Bias in Panel Data; Estimating the Return to Schooling," Annales de l'INSEE (April-September, 1978), 49-82.

(1982): "Multivariate Regression Models for Panel Data," Journal of Econometrics, 18, 5-46. (1984): "Panel Data," Chapter 22 in The Handbook of Econometrics, Vol. 2, ed. by Zvi Griliches and Michael Intrilligator. New York: North Holland. 
Duncan, Gary J., AND Daniel H. Hill (1985): “An Investigation of the Extent and Consequences of Measurement Error in Labor-Economic Survey Data," Journal of Labor Economics, 3, 508-532.

FreEman, Richard B. (1984): "Longitudinal Analysis of the Effects of Trade Unions," Journal of Labor Economics, 2, 1-26.

Fuller, Wayne, and T. C. Chua (1985): “Gross Change Estimation in the Presence of Response Error," Proceedings of the Conference on Gross Flows in Labor Force Statistics. Washington, D.C.: GPO, 65-77.

Ghez, Gilbert, And Gary S. Becker (1975): The Allocation of Time and Goods Over the Life Cycle. New York: Columbia University Press (for the National Bureau of Economic Research).

Grossman, Sanford, and Oliver D. Hart (1981): "Implicit Contracts, Moral Hazard and Unemployment," American Economic Review, 71, 301-307.

HALL, RoberT E. (1978): "Stochastic Implications of the Life-Cycle-Permanent Income Hypothesis: Theory and Evidence," Journal of Political Economy, 86, 971-987.

Hause, J. C. (1985): "The Fine Structure of Earnings and the On-the-Job Training Hypothesis," Econometrica, 52, 1013-1030.

HAUSMAN, JeRry (1978): “Specification Tests in Econometrics," Econometrica, 46, 1251-1271.

Heckman, James J. (1976): “A Life-Cycle Model of Earnings, Learning and Consumption,” Journal of Political Economy, 84, S11-S44.

Killingsworth, MARK (1983): Labor Supply. New York: Cambridge University Press.

Lillard, LeE A., AND YoRAM Weiss (1970): "Components of Variation in Panel Earnings Data: American Scientists 1960-1970," Econometrica, 47, 437-454.

MaCurdy, Thomas E. (1981): “An Empirical Model of Labor Supply in a Life-Cycle Setting," Journal of Political Economy, 89, 1059-1085.

- (1982): "The Use of Time Series Processes to Model the Error Structure of Earnings in a Longitudinal Data Analysis," Journal of Econometrics, 18, 83-114.

(1983): "A Simple Scheme for Estimating an Intertemporal Model of Labor Supply and Consumption in the Presence of Taxes and Uncertainty," International Economic Review, 24, 265-289.

Mellow, Wesley, AND Hal Sider (1983): “Accuracy of Response in Labor Market Surveys: Evidence and Implications," Journal of Labor Economics, 1, 331-344.

Newey, Whitney K. (1985): "Generalized Method of Moments Specification Tests," Journal of Econometrics, 29, 229-256.

Pencavel, John (1980): "Labor Supply of Men: A Survey," in The Handbook of Labor Economics, ed. by Orley Ashenfelter and Richard Layard. New York: North Holland.

Poterba, James M., and Lawrence H. Summers (1986): "Reporting Errors and Labor Market Dynamics," Econometrica, 54, 1319-1338.

SRI INTERNATIONAL (1983a): Seattle/Denver Income Maintenance Experiment. 1970:1978: Work Impact Record Report. Washington, D.C.: National Archive (microfiche M1363).

- (1983b): Final Report of the Seattle-Denver Income Maintenance Experiment: Volume I, Design and Results. Washington, D.C.: GPO.

Survey Research Center (1972): A Panel Study of Income Dynamics Procedures and Tape Codes Waves $I-V$. Ann Arbor, Michigan: Institute for Social Research, University of Michigan.

(1981): A Panel Study of Income Dynamics Procedures and Tape Codes 1980 Interview Year Wave XIII: A Supplement. Ann Arbor, Michigan: Institute for Social Research, University of Michigan.

Topel, Robert, AND Finis WelCh (1986): "Efficient Labor Contracts with Employment Risk," unpublished manuscript, University of Chicago Graduate School of Business. 
http://www.jstor.org

\section{LINKED CITATIONS}

- Page 1 of 8 -

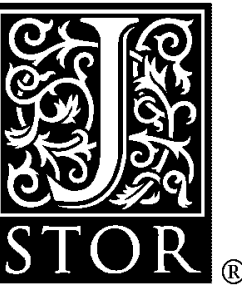

You have printed the following article:

On the Covariance Structure of Earnings and Hours Changes

John M. Abowd; David Card

Econometrica, Vol. 57, No. 2. (Mar., 1989), pp. 411-445.

Stable URL:

http://links.jstor.org/sici?sici=0012-9682\%28198903\%2957\%3A2\%3C411\%3AOTCSOE\%3E2.0.CO\%3B2-U

This article references the following linked citations. If you are trying to access articles from an off-campus location, you may be required to first logon via your library web site to access JSTOR. Please visit your library's website or contact a librarian to learn about options for remote access to JSTOR.

\section{[Footnotes]}

\footnotetext{
${ }^{2}$ An Empirical Model of Labor Supply in a Life-Cycle Setting

Thomas E. MaCurdy

The Journal of Political Economy, Vol. 89, No. 6. (Dec., 1981), pp. 1059-1085.

Stable URL:

http://links.jstor.org/sici?sici=0022-3808\%28198112\%2989\%3A6\%3C1059\%3AAEMOLS\%3E2.0.CO\%3B2-2

${ }^{2}$ Intertemporal Substitution in Labor Supply: Evidence from Micro Data

Joseph G. Altonji

The Journal of Political Economy, Vol. 94, No. 3, Part 2: Hoover Institution Labor Conference. (Jun., 1986), pp. S176-S215.

Stable URL:

http://links.jstor.org/sici?sici=0022-3808\%28198606\%2994\%3A3\%3CS176\%3AISILSE\%3E2.0.CO\%3B2-6
}

\footnotetext{
${ }^{3}$ A Life-Cycle Model of Earnings, Learning, and Consumption

James J. Heckman

The Journal of Political Economy, Vol. 84, No. 4, Part 2: Essays in Labor Economics in Honor of $\mathrm{H}$. Gregg Lewis. (Aug., 1976), pp. S11-S44.

Stable URL:

http://links.jstor.org/sici?sici=0022-3808\%28197608\%2984\%3A4\%3CS11\%3AALMOEL\%3E2.0.CO\%3B2-I
}

NOTE: The reference numbering from the original has been maintained in this citation list. 
http://www.jstor.org

\title{
LINKED CITATIONS
}

- Page 2 of 8 -

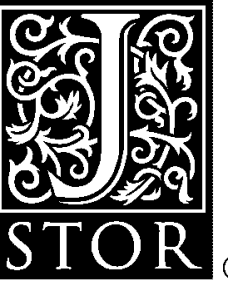

\footnotetext{
${ }^{3}$ A Profitable Approach to Labor Supply and Commodity Demands over the Life-Cycle Martin Browning; Angus Deaton; Margaret Irish

Econometrica, Vol. 53, No. 3. (May, 1985), pp. 503-544.

Stable URL:

http://links.jstor.org/sici?sici=0012-9682\%28198505\%2953\%3A3\%3C503\%3AAPATLS\%3E2.0.CO\%3B2-P

${ }^{5}$ Intertemporal Substitution in Labor Supply: Evidence from Micro Data

Joseph G. Altonji

The Journal of Political Economy, Vol. 94, No. 3, Part 2: Hoover Institution Labor Conference. (Jun., 1986), pp. S176-S215.

Stable URL:

http://links.jstor.org/sici?sici=0022-3808\%28198606\%2994\%3A3\%3CS176\%3AISILSE\%3E2.0.CO\%3B2-6
}

\author{
${ }^{6}$ An Empirical Model of Labor Supply in a Life-Cycle Setting \\ Thomas E. MaCurdy \\ The Journal of Political Economy, Vol. 89, No. 6. (Dec., 1981), pp. 1059-1085. \\ Stable URL: \\ http://links.jstor.org/sici?sici=0022-3808\%28198112\%2989\%3A6\%3C1059\%3AAEMOLS\%3E2.0.CO\%3B2-2
}

\section{${ }^{6}$ Intertemporal Substitution in Labor Supply: Evidence from Micro Data}

Joseph G. Altonji

The Journal of Political Economy, Vol. 94, No. 3, Part 2: Hoover Institution Labor Conference. (Jun., 1986), pp. S176-S215.

Stable URL:

http://links.jstor.org/sici?sici=0022-3808\%28198606\%2994\%3A3\%3CS176\%3AISILSE\%3E2.0.CO\%3B2-6

\section{${ }^{20}$ Accuracy of Response in Labor Market Surveys: Evidence and Implications}

Wesley Mellow; Hal Sider

Journal of Labor Economics, Vol. 1, No. 4. (Oct., 1983), pp. 331-344.

Stable URL:

http://links.jstor.org/sici?sici=0734-306X\%28198310\%291\%3A4\%3C331\%3AAORILM\%3E2.0.CO\%3B2-0

\section{${ }^{20}$ Longitudinal Analyses of the Effects of Trade Unions}

Richard B. Freeman

Journal of Labor Economics, Vol. 2, No. 1. (Jan., 1984), pp. 1-26.

Stable URL:

http://links.jstor.org/sici?sici=0734-306X\%28198401\%292\%3A1\%3C1\%3ALAOTEO\%3E2.0.CO\%3B2-H

NOTE: The reference numbering from the original has been maintained in this citation list. 
http://www.jstor.org

\section{LINKED CITATIONS}

- Page 3 of 8 -

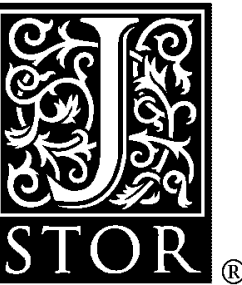

\section{${ }^{20}$ Estimating Gross Labor-Force Flows}

John M. Abowd; Arnold Zellner

Journal of Business \& Economic Statistics, Vol. 3, No. 3. (Jul., 1985), pp. 254-283.

Stable URL:

http://links.jstor.org/sici?sici=0735-0015\%28198507\%293\%3A3\%3C254\%3AEGLF\%3E2.0.CO\%3B2-D

\section{${ }^{20}$ An Investigation of the Extent and Consequences of Measurement Error in Labor-Economic Survey Data}

Greg J. Duncan; Daniel H. Hill

Journal of Labor Economics, Vol. 3, No. 4. (Oct., 1985), pp. 508-532.

Stable URL:

http://links.jstor.org/sici?sici=0734-306X\%28198510\%293\%3A4\%3C508\%3AAIOTEA\%3E2.0.CO\%3B2-R

\section{${ }^{20}$ Reporting Errors and Labor Market Dynamics}

James M. Poterba; Lawrence H. Summers

Econometrica, Vol. 54, No. 6. (Nov., 1986), pp. 1319-1338.

Stable URL:

http://links.jstor.org/sici?sici=0012-9682\%28198611\%2954\%3A6\%3C1319\%3AREALMD\%3E2.0.CO\%3B2-C

\section{${ }^{22}$ Specification Tests in Econometrics}

J. A. Hausman

Econometrica, Vol. 46, No. 6. (Nov., 1978), pp. 1251-1271.

Stable URL:

http://links.jstor.org/sici?sici=0012-9682\%28197811\%2946\%3A6\%3C1251\%3ASTIE\%3E2.0.CO\%3B2-X

\section{${ }^{24}$ A Life-Cycle Model of Earnings, Learning, and Consumption}

James J. Heckman

The Journal of Political Economy, Vol. 84, No. 4, Part 2: Essays in Labor Economics in Honor of H. Gregg Lewis. (Aug., 1976), pp. S11-S44.

Stable URL:

http://links.jstor.org/sici?sici=0022-3808\%28197608\%2984\%3A4\%3CS11\%3AALMOEL\%3E2.0.CO\%3B2-I

\section{${ }^{24}$ An Empirical Model of Labor Supply in a Life-Cycle Setting}

Thomas E. MaCurdy

The Journal of Political Economy, Vol. 89, No. 6. (Dec., 1981), pp. 1059-1085.

Stable URL:

http://links.jstor.org/sici?sici=0022-3808\%28198112\%2989\%3A6\%3C1059\%3AAEMOLS\%3E2.0.CO\%3B2-2

NOTE: The reference numbering from the original has been maintained in this citation list. 
http://www.jstor.org

\section{LINKED CITATIONS}

- Page 4 of 8 -

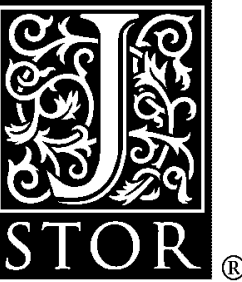

\footnotetext{
${ }^{24}$ Intertemporal Substitution in Labor Supply: Evidence from Micro Data

Joseph G. Altonji

The Journal of Political Economy, Vol. 94, No. 3, Part 2: Hoover Institution Labor Conference. (Jun., 1986), pp. S176-S215.

Stable URL:

http://links.jstor.org/sici?sici=0022-3808\%28198606\%2994\%3A3\%3CS176\%3AISILSE\%3E2.0.CO\%3B2-6
}

\section{${ }^{25}$ An Empirical Model of Labor Supply in a Life-Cycle Setting}

Thomas E. MaCurdy

The Journal of Political Economy, Vol. 89, No. 6. (Dec., 1981), pp. 1059-1085.

Stable URL:

http://links.jstor.org/sici?sici=0022-3808\%28198112\%2989\%3A6\%3C1059\%3AAEMOLS\%3E2.0.CO\%3B2-2

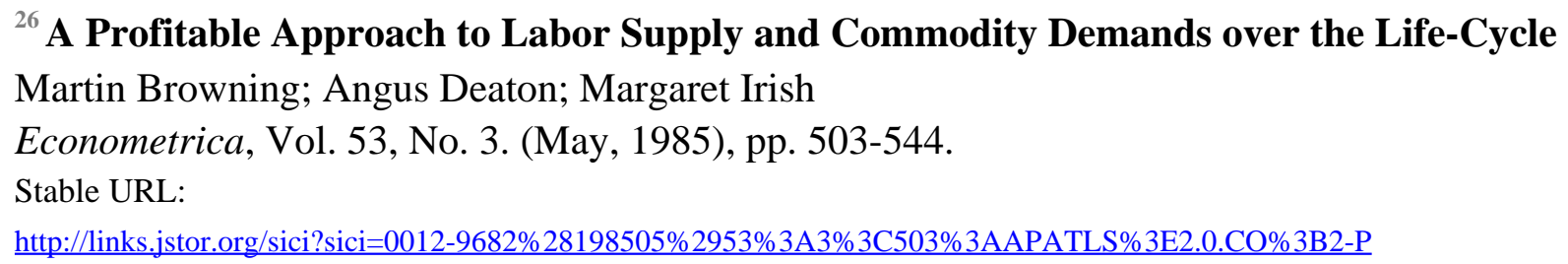

\section{${ }^{26}$ Stochastic Implications of the Life Cycle-Permanent Income Hypothesis: Theory and} Evidence

Robert E. Hall

The Journal of Political Economy, Vol. 86, No. 6. (Dec., 1978), pp. 971-987.

Stable URL:

http://links.jstor.org/sici?sici=0022-3808\%28197812\%2986\%3A6\%3C971\%3ASIOTLC\%3E2.0.CO\%3B2-K

\section{${ }^{27}$ Education, Unemployment, and Earnings}

Orley Ashenfelter; John Ham

The Journal of Political Economy, Vol. 87, No. 5, Part 2: Education and Income Distribution. (Oct., 1979), pp. S99-S116.

Stable URL:

http://links.jstor.org/sici?sici=0022-3808\%28197910\%2987\%3A5\%3CS99\%3AEUAE\%3E2.0.CO\%3B2-A

\section{${ }^{27}$ An Empirical Model of Labor Supply in a Life-Cycle Setting}

Thomas E. MaCurdy

The Journal of Political Economy, Vol. 89, No. 6. (Dec., 1981), pp. 1059-1085.

Stable URL:

http://links.jstor.org/sici?sici=0022-3808\%28198112\%2989\%3A6\%3C1059\%3AAEMOLS\%3E2.0.CO\%3B2-2

NOTE: The reference numbering from the original has been maintained in this citation list. 
http://www.jstor.org

\title{
LINKED CITATIONS
}

- Page 5 of 8 -

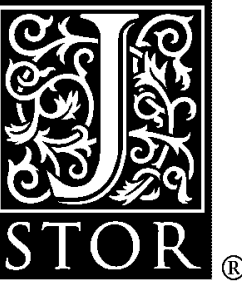

\footnotetext{
${ }^{27}$ A Profitable Approach to Labor Supply and Commodity Demands over the Life-Cycle Martin Browning; Angus Deaton; Margaret Irish

Econometrica, Vol. 53, No. 3. (May, 1985), pp. 503-544.

Stable URL:

http://links.jstor.org/sici?sici=0012-9682\%28198505\%2953\%3A3\%3C503\%3AAPATLS\%3E2.0.CO\%3B2-P

${ }^{28}$ A Simple Scheme for Estimating an Intertemporal Model of Labor Supply and Consumption in the Presence of Taxes and Uncertainty

Thomas E. MaCurdy

International Economic Review, Vol. 24, No. 2. (Jun., 1983), pp. 265-289.

Stable URL:

http://links.jstor.org/sici?sici=0020-6598\%28198306\%2924\%3A2\%3C265\%3AASSFEA\%3E2.0.CO\%3B2-S
}

\section{References}

\section{Intertemporal Labor Supply and Long-Term Employment Contracts}

John M. Abowd; David Card

The American Economic Review, Vol. 77, No. 1. (Mar., 1987), pp. 50-68.

Stable URL:

http://links.jstor.org/sici?sici=0002-8282\%28198703\%2977\%3A1\%3C50\%3AILSALE\%3E2.0.CO\%3B2-\%23

\section{Estimating Gross Labor-Force Flows}

John M. Abowd; Arnold Zellner

Journal of Business \& Economic Statistics, Vol. 3, No. 3. (Jul., 1985), pp. 254-283.

Stable URL:

http://links.jstor.org/sici?sici=0735-0015\%28198507\%293\%3A3\%3C254\%3AEGLF\%3E2.0.CO\%3B2-D

\author{
Intertemporal Substitution in Labor Supply: Evidence from Micro Data \\ Joseph G. Altonji \\ The Journal of Political Economy, Vol. 94, No. 3, Part 2: Hoover Institution Labor Conference. \\ (Jun., 1986), pp. S176-S215. \\ Stable URL: \\ http://links.jstor.org/sici?sici=0022-3808\%28198606\%2994\%3A3\%3CS176\%3AISILSE\%3E2.0.CO\%3B2-6
}

NOTE: The reference numbering from the original has been maintained in this citation list. 
http://www.jstor.org

\section{LINKED CITATIONS}

- Page 6 of 8 -

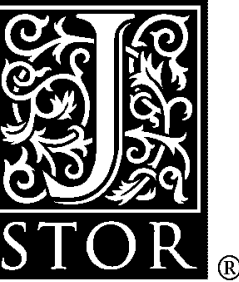

\section{Education, Unemployment, and Earnings}

Orley Ashenfelter; John Ham

The Journal of Political Economy, Vol. 87, No. 5, Part 2: Education and Income Distribution. (Oct., 1979), pp. S99-S116.

Stable URL:

http://links.jstor.org/sici?sici=0022-3808\%28197910\%2987\%3A5\%3CS99\%3AEUAE\%3E2.0.CO\%3B2-A

A Profitable Approach to Labor Supply and Commodity Demands over the Life-Cycle

Martin Browning; Angus Deaton; Margaret Irish

Econometrica, Vol. 53, No. 3. (May, 1985), pp. 503-544.

Stable URL:

http://links.jstor.org/sici?sici=0012-9682\%28198505\%2953\%3A3\%3C503\%3AAPATLS\%3E2.0.CO\%3B2-P

An Investigation of the Extent and Consequences of Measurement Error in Labor-Economic Survey Data

Greg J. Duncan; Daniel H. Hill

Journal of Labor Economics, Vol. 3, No. 4. (Oct., 1985), pp. 508-532.

Stable URL:

http://links.jstor.org/sici?sici=0734-306X\%28198510\%293\%3A4\%3C508\%3AAIOTEA\%3E2.0.CO\%3B2-R

\section{Longitudinal Analyses of the Effects of Trade Unions}

Richard B. Freeman

Journal of Labor Economics, Vol. 2, No. 1. (Jan., 1984), pp. 1-26.

Stable URL:

http://links.jstor.org/sici?sici=0734-306X\%28198401\%292\%3A1\%3C1\%3ALAOTEO\%3E2.0.CO\%3B2-H

\section{Implicit Contracts, Moral Hazard, and Unemployment}

Sanford J. Grossman; Oliver D. Hart

The American Economic Review, Vol. 71, No. 2, Papers and Proceedings of the Ninety-Third

Annual Meeting of the American Economic Association. (May, 1981), pp. 301-307.

Stable URL:

http://links.jstor.org/sici?sici=0002-8282\%28198105\%2971\%3A2\%3C301\%3AICMHAU\%3E2.0.CO\%3B2-W

Stochastic Implications of the Life Cycle-Permanent Income Hypothesis: Theory and Evidence Robert E. Hall

The Journal of Political Economy, Vol. 86, No. 6. (Dec., 1978), pp. 971-987.

Stable URL:

http://links.jstor.org/sici?sici=0022-3808\%28197812\%2986\%3A6\%3C971\%3ASIOTLC\%3E2.0.CO\%3B2-K

NOTE: The reference numbering from the original has been maintained in this citation list. 
http://www.jstor.org

\section{LINKED CITATIONS}

- Page 7 of 8 -

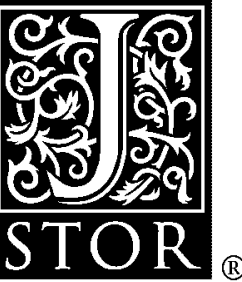

\section{Specification Tests in Econometrics}

J. A. Hausman

Econometrica, Vol. 46, No. 6. (Nov., 1978), pp. 1251-1271.

Stable URL:

http://links.jstor.org/sici?sici=0012-9682\%28197811\%2946\%3A6\%3C1251\%3ASTIE\%3E2.0.CO\%3B2-X

\section{A Life-Cycle Model of Earnings, Learning, and Consumption}

\section{James J. Heckman}

The Journal of Political Economy, Vol. 84, No. 4, Part 2: Essays in Labor Economics in Honor of H. Gregg Lewis. (Aug., 1976), pp. S11-S44.

Stable URL:

http://links.jstor.org/sici?sici=0022-3808\%28197608\%2984\%3A4\%3CS11\%3AALMOEL\%3E2.0.CO\%3B2-I

Components of Variation in Panel Earnings Data: American Scientists 1960-70

Lee A. Lillard; Yoram Weiss

Econometrica, Vol. 47, No. 2. (Mar., 1979), pp. 437-454.

Stable URL:

http://links.jstor.org/sici?sici=0012-9682\%28197903\%2947\%3A2\%3C437\%3ACOVIPE\%3E2.0.CO\%3B2-M

\section{An Empirical Model of Labor Supply in a Life-Cycle Setting}

Thomas E. MaCurdy

The Journal of Political Economy, Vol. 89, No. 6. (Dec., 1981), pp. 1059-1085.

Stable URL:

http://links.jstor.org/sici?sici=0022-3808\%28198112\%2989\%3A6\%3C1059\%3AAEMOLS\%3E2.0.CO\%3B2-2

\section{A Simple Scheme for Estimating an Intertemporal Model of Labor Supply and Consumption in the Presence of Taxes and Uncertainty}

Thomas E. MaCurdy

International Economic Review, Vol. 24, No. 2. (Jun., 1983), pp. 265-289.

Stable URL:

http://links.jstor.org/sici?sici=0020-6598\%28198306\%2924\%3A2\%3C265\%3AASSFEA\%3E2.0.CO\%3B2-S

Accuracy of Response in Labor Market Surveys: Evidence and Implications

Wesley Mellow; Hal Sider

Journal of Labor Economics, Vol. 1, No. 4. (Oct., 1983), pp. 331-344.

Stable URL:

http://links.jstor.org/sici?sici=0734-306X\%28198310\%291\%3A4\%3C331\%3AAORILM\%3E2.0.CO\%3B2-0

NOTE: The reference numbering from the original has been maintained in this citation list. 
http://www.jstor.org

\section{LINKED CITATIONS \\ - Page 8 of 8 -}

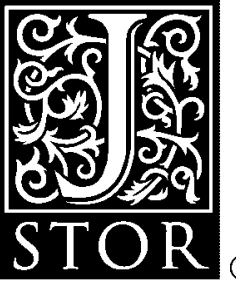

\section{Reporting Errors and Labor Market Dynamics}

James M. Poterba; Lawrence H. Summers

Econometrica, Vol. 54, No. 6. (Nov., 1986), pp. 1319-1338.

Stable URL:

http://links.jstor.org/sici?sici=0012-9682\%28198611\%2954\%3A6\%3C1319\%3AREALMD\%3E2.0.CO\%3B2-C

NOTE: The reference numbering from the original has been maintained in this citation list. 